\title{
The Wadge order on the Scott domain is not a well-quasi-order
}

\author{
JACQUes DuparC ${ }^{1}$ AND Louis Vuilleumier ${ }^{* 1,2}$ \\ ${ }^{1}$ Department of Information Systems (DESI), \\ University of Lausanne, Switzerland \\ JacQues . Duparc@unil. Ch, Louis. Vui lleumier. 1@unil . ch \\ ${ }^{2}$ Research Institute on the Foundations of Computer Science (IRIF), \\ Paris Diderot University, Sorbonne Paris Cité, France \\ LOUIS. VuI LLEUMIER@ETU. UNIV-PARIS-DIDEROT . FR
}

\begin{abstract}
We prove that the Wadge order on the Borel subsets of the Scott domain is not a well-quasi-order, and that this feature even occurs among the sets of Borel rank at most 2. For this purpose, a specific class of countable 2-colored posets $\mathbb{P}_{\text {emb }}$ equipped with the order induced by homomorphisms is embedded into the Wadge order on the $\boldsymbol{\Delta}_{2}^{0}$-degrees of the Scott domain. We then show that $\mathbb{P}_{\text {emb }}$ both admits infinite strictly decreasing chains and infinite antichains with respect to this notion of comparison, which therefore transfers to the Wadge order on the $\boldsymbol{\Delta}_{2^{-}}^{0}$ degrees of the Scott domain.

Keywords: quasi-Polish space, Scott domain, Wadge reducibility, wellquasi-order.

Mathematics Subject Classification: 03B70, 03D30, 03E15, 54H05, 68Q15.
\end{abstract}

With the exception of Section 5, all the results presented in this article including the main ones - are due to the sole second author.

\section{Introduction}

The Wadge order $\leq_{w}$ - named after Wadge Wad83 - on the subsets of a topological space $X$ is the quasi-order induced by reductions via continuous functions. More precisely, if $A, B \subseteq X$, then $A \leq_{w} B$ if there exists a continuous function $f: X \rightarrow X$ such that $f^{-1}[B]=A$, i.e., $x \in A \Leftrightarrow f(x) \in B$ for all $x \in X$. The Wadge order measures the topological complexity of the subsets of $X$. Indeed, $A \leq_{w} B$ means that the membership problem for $A$ can be reduced, via some continuous function, to the membership problem for $B$; or, in other words, $A$ is topologically less complicated than $B$.

The Wadge order is a refinement of both the classical Borel and HausdorffKuratowski difference hierarchies since when $B$ is located strictly higher than

*The second author gratefully acknowledges support from the Swiss National Science Foundation grant 200021-159241. 
$A$ in one of these hierarchies, then $A \leq_{w} B$ holds. Over the last 50 years, this quasi-order has been extensively studied in the context of Polish spaces - i.e., the separable completely metrizable spaces [AL12, And07, Dup01, Ike10, Kec95, KM17, Lou12, LSR12, Sch18, VW12, Wad83, Wad12].

Over the last decades, some slightly different classes of topological spaces rose interest for their involvement in computer science $\mathrm{GHK}^{+} 03, \mathrm{GL13}, \mathrm{Sco76}$, Sel05, Sel06, Wei00. This has been the case, in particular, of non-metrizable hence non-Polish - spaces occurring as domains of the semantic of programming languages. Building on a prior work of Selivanov - that extensively studied a generalized version of the Borel hierarchy to non-metrizable spaces [Sel05, Sel06] - de Brecht introduced in dB13 the class of quasi-Polish spaces - i.e., the second countable quasi-metrizable spaces, where a quasi-metric is a metric whose symmetry condition has been dropped. In particular, de Brecht proved that some of the major results of descriptive set theory extend to quasi-Polish spaces (see Theorems 19, 23, 58 and 70 in [dB13]). He also exhibited the Scott domain 1 $\mathcal{P} \omega$ as a universal quasi-Polish space. More precisely, de Brecht proved that the quasi-Polish spaces are - up to homeomorphism - exactly the $\Pi_{2}^{0}$-subsets of $\mathcal{P} \omega$ (Theorem 24 in $\mathrm{dB13}$ ), where $\mathcal{P} \omega$ is the power set of the integers equipped with the topology where a basic open set is composed of all the sets that contain a fixed finite subset of the integers.

More results by de Brecht suggest that a reasonable descriptive set theory still holds in the quasi-Polish setting. Unfortunately, very few is known about the Wadge order in this context. To the contrary, the Polish spaces $X$ whose Wadge order on the Borel subsets is well-founded and contains no infinite antichain - or in other words, $\leq_{w}$ is a well-quasi-order on the Borel subsets of $X-$ were recently characterized in [Sch18] as the zero-dimensional ones - i.e., Polish spaces admitting a clopen basis. Whether this result generalizes to quasi-Polish spaces remains open. In a first attempt to tackle this question, we propose to study the Wadge order on the subsets of the Scott domain $\mathcal{P} \omega$.

Several results have already been obtained by Selivanov who proved the existence of $\leq_{w}$-antichains of size 4 for $\mathcal{P} \omega$, as well as the existence of $\leq_{w^{-}}$ minimal sets at each level of the difference hierarchy of open sets [Sel05]; and by Becher and Grigorieff who exhibited, for each infinite level $\alpha$ of the difference hierarchy of open sets, some strictly $\leq_{w}$-increasing chains of sets of length $\alpha$, and also described the $\leq_{w}$-maximal sets for each such level for a large number of quasi-Polish spaces including $\mathcal{P} \omega$ [BG15]. In this article, we show both that the Wadge order on the subsets of $\mathcal{P} \omega$ is ill-founded and that it admits infinite antichains. Moreover, we show that these properties occur already within the differences of $\omega$ open sets, i.e., at a very low level of topological complexity:

Theorem 36. $\left(D_{\omega}\left(\boldsymbol{\Sigma}_{1}^{0}\right)(\mathcal{P} \omega), \leq_{w}\right)$ is ill-founded.

Theorem 40. $\left(D_{\omega}\left(\boldsymbol{\Sigma}_{1}^{0}\right)(\mathcal{P} \omega), \leq_{w}\right)$ has infinite antichains.

These results are obtained through a generalization of a construction introduced by Selivanov in Sel05]. More precisely, we define an order-embedding from a class of 2-colored countable posets $\mathbb{P}_{\text {emb }}$ (Definition [16) endowed with the usual notion of comparison by homomorphisms into the Wadge order on the $\Delta_{2}^{0}$-degrees of $\mathcal{P} \omega$, where a degree is an equivalence class induced by $\leq_{w}$ :

\footnotetext{
${ }^{1}$ The Scott domain was first introduced by Scott as a denotational semantic for the $\lambda$ calculus $\mathrm{Sco} 76$.
} 
Theorem 26. There exists an order-embedding:

$$
\left(\mathbb{P}_{\mathrm{emb}}, \preccurlyeq_{c}\right) / \equiv_{c} \rightarrow\left(\boldsymbol{\Delta}_{2}^{0}(\mathcal{P} \omega), \leq_{w}\right) / \equiv_{w} .
$$

Different approaches have already been considered for tackling the problem of classifying subsets of non-Polish spaces according to their topological complexity. For instance, Pequignot studied the quasi-order obtained from reductions via admissible representations Peq15, and Motto Ros, Schlicht and Selivanov investigated the quasi-order obtained from classes of reductions that are larger than the continuous ones [MRSS15].

The article is organized as follows. We fix notations and general definitions in Section 2, where we also recall results such as the characterizations of some of the topological classes obtained by Selivanov in Sel05]. In Section 3, we define the class of posets $\mathbb{P}_{\text {emb }}$ (Definition 16) that we embed into the Wadge order of $\mathcal{P} \omega$ (Theorem 26) in Section 4. This order-embedding is the main construction of this article. A game characterization of reductions between 2-colored posets is introduced in Section 5 (Definition 27) in order to show, in Section 6 and 7, that the Wadge order of $\mathcal{P} \omega$ is ill-founded (Theorem 36 ) and that it has infinite antichains (Theorem 40). We conclude in Section 8 with open questions.

\section{Preliminaries}

\subsection{General notations}

As usual, we denote by $\omega$ or $\mathbb{N}$ the set of all integers and by $\aleph_{0}$ its cardinality. We also write $\omega^{+}$for $\omega \backslash\{0\}$ and $\omega_{1}$ for the first uncountable ordinal. We use the letters $i, j, k, l, m, n$ for integers and $\alpha, \beta, \gamma$ for arbitrary ordinals. Since every ordinal is regarded as the set of its predecessors, if $n \in \omega$, the notation $x \cap n$ stands for $x \cap\{0,1, \ldots, n-1\}$.

Given any sets $X, Y$, if $f: X \rightarrow Y$ is a function, $A \subseteq X$, and $B \subseteq Y$, then we write $f[A]=\{f(x) \mid x \in A\}$ and $f^{-1}[B]=\{x \mid f(x) \in B\}$. If $f$ is injective, we write $f^{-1}(y)$ for the unique element $x \in X$ such that $f(x)=y$.

An $X$-sequence - or simply a sequence - is a function $s: \alpha \rightarrow X$-denoted by $\left(s_{\beta}\right)_{\beta<\alpha}$ - from some ordinal $\alpha$ called the length of the sequence to $X$. In this article, we will mainly consider sequences such that $\alpha \in \omega+1=\omega \cup\{\omega\}$. We use the letters $s, t$ to denote sequences. The only sequence of length 0 - the empty sequence - is denoted by $\varnothing$. If $s, t$ are sequences, then $t$ is a prefix of $s$, written $t \sqsubseteq s$, if $\ln (t) \leq \ln (s)$ and $s_{k}=t_{k}$ for all $k<\ln (t)$. If $t \sqsubseteq s$ but $s \nsubseteq t$, we write $t \sqsubset s$. If $s, t$ are $X$-sequences, the concatenation of $s$ and $t$ is defined by $s \frown t=\left(s_{0}, \ldots, s_{\operatorname{lh}(s)-1}, t_{0}, \ldots, t_{\operatorname{lh}(t)-1}\right)$. The set of all $X$-sequences of finite length is denoted by $X^{<\omega}$.

A tree $T \subseteq X^{<\omega}$ is a set of finite $X$-sequences closed under the prefix relation 2. It is well-founded if it has no infinite branch 3 , in which case the rank of any $t \in T$ is (well-)defined by $\sqsupseteq$-induction: $\operatorname{rk}_{T}(t)=0$ if $t$ is $\sqsubseteq$-maximal and $\operatorname{rk}_{T}(t)=\sup \left\{\operatorname{rk}_{T}(s)+1 \mid t \sqsubset s\right\}$ otherwise. The $\operatorname{rank} \operatorname{rk}(T)$ of a non-empty well-founded tree $T$ is the ordinal $\operatorname{rk}_{T}(\varnothing)$.

\footnotetext{
${ }^{2}$ If $t \in T$ and $s \sqsubseteq t$, then $s \in T$.

${ }^{3}$ An infinite branch is a function $f: \omega \rightarrow T$ such that, if $n<m$, then $f(n) \sqsubset f(m)$.
} 


\section{$2.2 \quad$ Order-theoretic notations}

A quasi-order on a set $Q$ is any reflexive and transitive relation $4 \leq_{q} \subseteq Q \times Q$. Whenever $\leq_{q}$ is clear from the context, we write $Q$ for the couple $\left(Q, \leq_{q}\right)$. We will use the letters $P, Q$ for quasi-orders and $p \in P, q \in Q$ for their elements. As usual, $q_{0} \leq_{q} q_{1}$ stands for $\left(q_{0}, q_{1}\right) \in \leq_{q}$, and $q_{0}<_{q} q_{1}$ for $q_{0} \leq_{q} q_{1}$ but $q_{1} \leq_{q} q_{0}$. If $q_{0} \varliminf_{q} q_{1}$ and $q_{1} \Varangle_{q} q_{0}$, then $q_{0}$ and $q_{1}$ are said to be incomparable which is denoted by $q_{0} \perp_{q} q_{1}$. If $Q$ is a quasi-order and $P \subseteq Q$, then $P$ equipped with the induced relation is a quasi-order. An infinite antichain in $Q$ is a sequence $\left(q_{n}\right)_{n<\omega}$ of pairwise incomparable elements, and a strictly $\leq_{q}$-increasing (resp. strictly $\leq_{q}$-decreasing) sequence is a sequence $\left(q_{n}\right)_{n<\omega}$ such that $q_{n}<_{q} q_{n+1}$ (resp. $q_{n+1}<_{q} q_{n}$ ) for all $n \in \omega$. A well-quasi-order is a quasi-order $Q$ that has no infinite antichain and no strictly $\leq_{q}$-decreasing sequence. We denote by $\operatorname{Pred}(q)=\left\{q^{\prime} \in Q \mid q^{\prime} \leq_{q} q\right\}$ the set of predecessors of $q \in Q$, and by $\operatorname{Pred}_{\mathrm{im}}(q)=\left\{q^{\prime} \in Q \mid\left(q^{\prime}<_{q} q\right) \wedge \neg \exists q^{\prime \prime} \in Q\left(q^{\prime}<_{q} q^{\prime \prime} \wedge q^{\prime \prime}<_{q} q\right)\right\}$ the set of its immediate predecessors.

We use homomorphisms 5 in order to compare structures. If there exists an injective homomorphism $\varphi: P \rightarrow Q$, then we write $P \stackrel{1-1 h}{\longrightarrow} Q$; if it is injective and preserves immediate predecessor 6 , then we write $P \longmapsto Q$. Notice that $P \longmapsto Q$ is more rigid than $P \stackrel{1-1 h .}{\longrightarrow} Q$, hence, it describes more local behaviors.

If $q$ and $q^{\prime}$ are elements of a quasi-order $Q$ such that $q \leq_{q} q^{\prime}$ and $q^{\prime} \leq_{q} q$, then we write $q \equiv_{q} q^{\prime}$. The relation $\equiv_{q}$ is an equivalence relation whose equivalence classes are denoted by $[q]=\left\{q^{\prime} \in Q \mid q \equiv_{q} q^{\prime}\right\}$. The quotient set $Q / \equiv_{q}=\{[q] \mid$ $q \in Q\}$ inherits the quasi-order $\leq_{q}$. More precisely, we set $[q] \leq_{q}\left[q^{\prime}\right]$ if and only if $q \leq q^{\prime}$. The set $Q / \equiv_{q}$ equipped with $\leq_{q}$ is a poset, i.e., a quasi-order whose order-relation is a partial order 7 .

We denote the class of countable posets by $\mathbb{P}$. If $P \in \mathbb{P}$, then we can always consider $\leq_{p} \subseteq \alpha \times \alpha$ where $\alpha \in \omega \cup\{\omega\}$ via any bijection: $P \leftrightarrow \alpha$; so that all the posets we consider are posets on $P \in \omega \cup\{\omega\}$. An order-embedding is a homomorphism between two posets $\varphi: P \rightarrow Q$ such that for any $p_{0}, p_{1} \in P$, $p_{0} \leq_{p} p_{1}$ if and only if $\varphi\left(p_{0}\right) \leq_{q} \varphi\left(p_{1}\right)$. Thus, order-embeddings are injective. The main posets studied in this article will be the set of finite subsets of the integers ordered by inclusion $\left(\mathcal{P}_{<\omega}(\omega), \subseteq\right)$, and the set of infinite subsets of the integers ordered by inclusion $\left(\mathcal{P}_{\omega}(\omega), \subseteq\right)$.

A 2-colored poset is a triple $\mathrm{P}=\left(P, \leq_{p}, \mathrm{c}_{p}\right)$ where $\leq_{p}$ is a partial order on $P$ and $c_{p}: P \rightarrow 2$ is a 2 -coloring. We usually use the letters $\mathrm{P}, \mathrm{Q}$ for 2 colored posets. We also compare them via homomorphisms 8 . If there exists a homomorphism from $\mathrm{P}$ to $\mathrm{Q}$, then we write $\mathrm{P} \preccurlyeq_{c} \mathrm{Q}$; if this homomorphism is injective, then we write $\mathrm{P} \stackrel{1-1 h .}{\longrightarrow} \mathrm{Q}$; if it is injective and preserves immediate predecessors, then we write $\mathrm{P} \longmapsto_{c} \mathrm{Q}$. Notice that $\preccurlyeq_{c}$ is a quasi-order on 2-colored posets. We will denote by $\equiv_{c}$ the induced equivalence relation.

\footnotetext{
${ }^{4} \mathrm{~A}$ binary relation $\leq_{q}$ on $Q$ is reflexive if, for all $q \in Q,(q, q) \in \leq_{q}$, and transitive if, for any $q_{0}, q_{1}, q_{2} \in Q,\left(q_{0}, q_{1}\right),\left(q_{1}, q_{2}\right) \in \leq_{q}$ implies $\left(q_{0}, q_{2}\right) \in \leq_{q}$.

${ }^{5} \mathrm{~A}$ homomorphism between two quasi-orders $P$ and $Q$ is a function $\varphi: P \rightarrow Q$ such that for any $p_{0}, p_{1} \in P$, if $p_{0} \leq_{p} p_{1}$, then $\varphi\left(p_{0}\right) \leq_{q} \varphi\left(p_{1}\right)$.

${ }^{6} \mathrm{~A}$ function $\varphi: P \rightarrow Q$ preserves immediate predecessors if, for any $p_{0}, p_{1} \in P$, whenever $p_{0} \in \operatorname{Pred}\left(p_{1}\right)$, then $\varphi\left(p_{0}\right) \in \operatorname{Pred}\left(\varphi\left(p_{1}\right)\right)$.

${ }^{7}$ A quasi-order $\left(P, \leq_{p}\right)$ is a partial order if $\leq_{p}$ is antisymmetric, i.e., for any $p_{0}, p_{1} \in P$, $p_{0} \leq_{p} p_{1}$ and $p_{1} \leq_{p} p_{0}$ implies $p_{0}=p_{1}$.

${ }^{8} \mathrm{~A}$ homomorphism between $\mathrm{P}, \mathrm{Q}$ two 2-colored posets is a quasi-order homomorphism $\varphi: P \rightarrow Q$ such that for all $p \in P, \mathrm{c}_{p}(p)=\mathrm{c}_{q}(\varphi(p))$.
} 


\subsection{Topological notations}

This article focuses on the study of a particular topological space first introduced by Scott as a universal model of the semantic of $\lambda$-calculus [Sco76].

Definition 1. The Scott domain is the power set of the integers $\mathcal{P}(\omega)$ equipped with the topology generated by the basis

$$
\left\{\mathcal{O}_{F} \mid F \in \mathcal{P}_{<\omega}(\omega)\right\} \text {, where } \mathcal{O}_{F}=\{x \subseteq \omega \mid F \subseteq x\} .
$$

The Scott domain is a non-metrizable - in fact non-Hausdorff $\left(T_{2}\right)$, and even non-Fréchet $\left(T_{1}\right)$ - compact space which is connected and Kolmogorov $\left(T_{0}\right)$.

From now on and throughout this article, we use the notation $\mathcal{P} \omega$ for the Scott domain; $F, G, H$ for finite subsets of $\omega ; x, y, z$ for arbitrary subsets of $\omega$; and $\mathcal{A}, \mathcal{B}, \mathcal{C}$ for subsets of $\mathcal{P} \omega$.

Our ultimate goal is to study the topological complexity of subsets of $\mathcal{P} \omega$. In metrizable spaces, this study begins with the definition of the Borel hierarchy (Section 11.B in Kec95). However, the same construction would not work with $\mathcal{P} \omega$ for it is not metrizable. To overcome this obstacle, Selivanov introduced a new version of the Borel hierarchy for arbitrary spaces [Sel05, Sel06]. This generalization extends the original one and induces a well-behaved hierarchy (see $\mathrm{dB} 13$ for more details). In the rest of this section, $\mathcal{T}$ denotes a topology on a set $X$. As usual, we denote by $X$ both the topological space and the underlying set without any risk of confusion.

Definition 2. We define $\boldsymbol{\Sigma}_{1}^{0}(X)=\mathcal{T}$, and for $1<\alpha<\omega_{1}$,

$$
\Sigma_{\alpha}^{0}(X)=\left\{\bigcup_{n \in \omega}\left(B_{n} \backslash B_{n}^{\prime}\right) \mid B_{n}, B_{n}^{\prime} \in \Sigma_{\beta_{n}}^{0}(X), \beta_{n}<\alpha\right\} .
$$

We also define $\boldsymbol{\Pi}_{\alpha}^{0}(X)=\left\{A \subseteq X \mid X \backslash A \in \boldsymbol{\Sigma}_{\alpha}^{0}(X)\right\}, \boldsymbol{\Delta}_{\alpha}^{0}(X)=\boldsymbol{\Sigma}_{\alpha}^{0}(X) \cap \boldsymbol{\Pi}_{\alpha}^{0}(X)$ for $\alpha<\omega_{1}$. Finally, we define the Borel sets as $\mathcal{B}(X)=\bigcup_{\alpha \in \omega_{1}} \boldsymbol{\Sigma}_{\alpha}^{0}(X)$.

The Borel hierarchy on $X$ is the quasi-order

$$
\left(\left\{\boldsymbol{\Sigma}_{\alpha}^{0}(X), \boldsymbol{\Pi}_{\alpha}^{0}(X)\right\}_{\alpha \in \omega_{1}}, \subseteq\right)
$$

As customary in descriptive set theory, we consider the Hausdorff-Kuratowski difference hierarchy as a first refinement of the Borel hierarchy (see Section 22.E in [Kec95]). Its definition relies on the difference operation.

Definition 3. If $0<\alpha<\omega_{1}$ and $\left(A_{\beta}\right)_{\beta<\alpha}$ is a sequence of subsets of $X$, then

$$
D_{\alpha}\left(\left(A_{\beta}\right)_{\beta<\alpha}\right)=\bigcup\left\{\begin{array}{l|l}
A_{\beta} \backslash \cup_{\gamma<\beta} A_{\gamma} & \begin{array}{l}
\beta<\alpha, \text { and } \\
\alpha \text { and } \beta \text { have different parities }
\end{array}
\end{array}\right\} \subseteq X .
$$

If $0<\alpha, \beta<\omega_{1}$, then

$$
D_{\alpha}\left(\boldsymbol{\Sigma}_{\beta}^{0}\right)(X)=\left\{D_{\alpha}\left(\left(A_{\gamma}\right)_{\gamma<\alpha}\right) \mid\left(A_{\gamma}\right)_{\gamma<\alpha} \subseteq \boldsymbol{\Sigma}_{\beta}^{0}(X)\right\} \subseteq \mathcal{P}(X) .
$$

Finally, we set $\check{D}_{\alpha}\left(\boldsymbol{\Sigma}_{\beta}^{0}\right)(X)=\left\{A \subseteq X \mid X \backslash A \in D_{\alpha}\left(\boldsymbol{\Sigma}_{\beta}^{0}\right)(X)\right\}$.

The Hausdorff-Kuratowski difference hierarchy on $X$ is the quasi-order

$$
\left(\left\{D_{\alpha}\left(\boldsymbol{\Sigma}_{\beta}^{0}\right)(X), \check{D}_{\alpha}\left(\boldsymbol{\Sigma}_{\beta}^{0}\right)(X)\right\}_{\alpha, \beta \in \omega_{1}}, \subseteq\right) .
$$


All Borel and Hausdorff-Kuratowski classes previously defined are closed under continuous preimages9. This suggests a natural further investigation of topological complexity through the lens of Wadge reducibility, a notion of comparison first studied thoroughly by Wadge in his PhD thesis Wad83.

Definition 4. Let $A, B \subseteq X$. The set $A$ is Wadge reducible to $B$, written $A \leq_{w} B$, if there exists a continuous function $f: X \rightarrow X$ such that for all $x \in X$,

$$
x \in A \Longleftrightarrow f(x) \in B
$$

i.e., $f^{-1}[B]=A$.

$A$ is Wadge equivalent to $B$, written $A \equiv_{w} B$, if $A \leq_{w} B$ and $B \leq_{w} A$ hold.

Since both the identity and the composition of continuous functions are continuous, $\leq_{w}$ induces a quasi-order on the subset of $X$, and thus the binary relations $<_{w}$, $\leq_{w}$ and $\perp_{w}$ are well-defined.

Definition 5. Let $X$ be any topological space and $\Gamma(X) \subseteq \mathcal{P}(X)$ be any class closed under continuous preimages. The Wadge order on the $\Gamma$-subsets of $X$ is the quasi-order $\left(\Gamma(X), \leq_{w}\right)$.

For the equivalence relation $\equiv_{w}$, we have a special terminology:

Definition 6. Let $X$ be any topological space, $A \subseteq X$ and $\Gamma(X) \subseteq \mathcal{P}(X)$ be any class closed under continuous preimages.

The Wadge degree of $A$ is its $\equiv_{w}$-equivalence class $[A]=\left\{B \subseteq A \mid A \equiv_{w} B\right\}$.

The Wadge order on the $\Gamma$-degrees of $X$ is the poset $\left(\Gamma(X), \leq_{w}\right) / \equiv_{w}$.

\subsection{Selivanov's toolbox}

We will restrict ourselves to the study of the quasi-order $\left(\boldsymbol{\Delta}_{2}^{0}(\mathcal{P} \omega), \leq_{w}\right)$. As mentioned in the Introduction, some results have already been obtained on this quasi-order in Sel05 and BG15. The main result of this article (Theorem 26) comes as a generalization of a construction introduced by Selivanov in Sel05 that we recall here.

Definition 7 (pp.56 in Sel05). Let $T_{\alpha}$ be any well-founded tree of rank $\omega \leq$ $\alpha<\omega_{1}, \xi: \omega^{<\omega} \rightarrow \omega$ be any injective mapping such that $\xi(\varnothing)=0$, and $e: T_{\alpha} \rightarrow \mathcal{P}_{<\omega}(\omega)$ be defined as $e(s)=\{\xi(t) \mid t \sqsubseteq s\}$. The sets $Y_{\alpha}$ and $Z_{\alpha}$ are defined by:

1. $Y_{\alpha}=e\left[T_{\alpha}^{1}\right]$, where $T_{\alpha}^{1}=\left\{s \in T_{\alpha} \mid \operatorname{lh}(s)\right.$ is odd $\}$,

2. $Z_{\alpha}=B\left(T_{\alpha}\right) \cup Y_{\alpha}$, where $B\left(T_{\alpha}\right)=\left\{x \subseteq \omega \mid \forall s \in T_{\alpha} x \nsubseteq e(s)\right\}$.

In Sel05], it is shown that, given any $\omega \leq \alpha<\omega_{1}, Y_{\alpha}$ and $Z_{\alpha}$ are differences of $\alpha$ open sets, Wadge incomparable, and $\leq_{w}$-minimal among true differences of $\alpha$ open sets. More precisely:

Theorem 8 (Propositions 5.9 and 6.4 in Sel05]). For $n \in \omega, \omega \leq \alpha, \beta<\omega_{1}$ and $\mathcal{A} \in \boldsymbol{\Delta}_{2}^{0}(\mathcal{P} \omega) \backslash \check{D}_{\alpha}\left(\boldsymbol{\Sigma}_{1}^{0}\right)(\mathcal{P} \omega)$, we have:

1. $D_{n}\left(\boldsymbol{\Sigma}_{1}^{0}\right)(\mathcal{P} \omega) \backslash \check{D}_{n}\left(\boldsymbol{\Sigma}_{1}^{0}\right)(\mathcal{P} \omega)$ and $\check{D}_{n}\left(\boldsymbol{\Sigma}_{1}^{0}\right)(\mathcal{P} \omega) \backslash D_{n}\left(\boldsymbol{\Sigma}_{1}^{0}\right)(\mathcal{P} \omega)$ form two incomparable Wadge degrees,

\footnotetext{
${ }^{9} \mathrm{~A}$ class of subsets $\Gamma(X) \subseteq \mathcal{P}(X)$ is closed under continuous preimages if for any $A \in$ $\Gamma(X), f: X \rightarrow X$ continuous, then $f^{-1}[A] \in \Gamma(X)$.
} 
2. $Y_{\alpha}, Z_{\alpha} \in D_{\alpha}\left(\boldsymbol{\Sigma}_{1}^{0}\right)(\mathcal{P} \omega) \backslash \check{D}_{\alpha}\left(\boldsymbol{\Sigma}_{1}^{0}\right)(\mathcal{P} \omega)$,

3. $Y_{\alpha} \perp_{w} Z_{\beta}$,

4. if $\omega \in \mathcal{A}$, then $Z_{\alpha} \leq_{w} \mathcal{A}$,

5. if $\omega \notin \mathcal{A}$, then $Y_{\alpha} \leq_{w} \mathcal{A}$.

The proof of Theorem 8 makes use of Selivanov's characterizations of the $\boldsymbol{\Delta}_{2}^{0}$-subsets and of the $D_{\alpha}\left(\boldsymbol{\Sigma}_{1}^{0}\right)$-subsets of $\mathcal{P} \omega$. Since our proof will also require these characterizations, we first recall them. For this purpose, if $x, y \in \mathcal{P} \omega$ such that $x \subseteq y$, we introduce the notation

$$
[x, y]=\{z \in \mathcal{P} \omega \mid x \subseteq z \subseteq y\} .
$$

Definition 9 (Definition 2.4 in Sel05]). $\mathcal{A} \subseteq \mathcal{P} \omega$ is approximable if, for all $x \in \mathcal{A}$, there exists $F \in \mathcal{P}_{<\omega}(\omega)$ such that $F \subseteq x$ and $[F, x] \subseteq \mathcal{A}$.

A subset $\mathcal{A}$ of $\mathcal{P} \omega$ is $\boldsymbol{\Delta}_{2}^{0}$ if the membership of any subset $x \subseteq \omega$ to $\mathcal{A}$ can be approximated by a finite subset of $x$. More precisely:

Theorem 10 (Theorem 3.12 in Sel05]). Let $\mathcal{A} \subseteq \mathcal{P} \omega$.

$$
\mathcal{A} \in \boldsymbol{\Delta}_{2}^{0}(\mathcal{P} \omega) \Longleftrightarrow \mathcal{A} \text { and } \mathcal{P} \omega \backslash \mathcal{A} \text { are approximable. }
$$

The characterization of $D_{\alpha}\left(\Sigma_{1}^{0}\right)$-subsets of $\mathcal{P} \omega$ is a stratification of the previous result using the notion of a 1-alternating tree.

Definition 11 (Definition 3.5 in Sel05]). Let $\mathcal{A} \subseteq \mathcal{P} \omega$ and $0<\alpha<\omega_{1}$. A 1-alternating tree for $\mathcal{A}$ of rank $\alpha$ is a homomorphism of quasi-orders

$$
f:(T, \sqsubseteq) \rightarrow\left(\mathcal{P}_{<\omega}(\omega), \subseteq\right)
$$

from a well-founded tree $T \subseteq \omega^{<\omega}$ of rank $\alpha$ such that:

1. $f(\varnothing) \in \mathcal{A}$, and

2. for all $s \frown\langle n\rangle \in T$, we have $(f(s) \in \mathcal{A} \leftrightarrow f(s \frown\langle n\rangle) \notin \mathcal{A})$.

Corollary 12 (Corollary 3.11 in Sel05]). Let $\mathcal{A} \subseteq \mathcal{P} \omega$ and $0<\alpha<\omega_{1}$.

$$
\mathcal{A} \in D_{\alpha}\left(\boldsymbol{\Sigma}_{1}^{0}\right)(\mathcal{P} \omega) \Longleftrightarrow\left\{\begin{array}{c}
\mathcal{A} \in \boldsymbol{\Delta}_{2}^{0}(\mathcal{P} \omega) \text { and } \\
\text { there is no 1-alternating tree for A of rank } \alpha
\end{array}\right.
$$

\section{The class $\mathbb{P}_{\text {emb }}$}

We define a class - called $\mathbb{P}_{\mathrm{emb}}$ - of countable 2-colored posets (Definition 14) that will be mapped into the Wadge order on the subsets of the Scott domain in the next section. The definition of $\mathbb{P}_{\text {emb }}$ will first be independent of $\mathcal{P} \omega$. Afterwards, we will give an order theoretic characterization of the elements of $\mathbb{P}_{\text {emb }}$ that link them to $\mathcal{P} \omega$ (Proposition 15 ).

We begin with the naming of several posets that are useful for the definition of a subclass of $\mathbb{P}$ denoted by $\mathbb{P}_{\text {shr }}$. In the following picture, we represent each poset $\left(P, \leq_{p}\right)$ with a directed graph $G=(P, \rightarrow)$. More precisely, if $p, q \in P$, then $p \leq_{p} q$ if and only if there exists a finite sequence $\left(p_{k}\right)_{k \leq l}$ such that $p_{0}=p, p_{l}=q$ and for all $k<l$, we have $p_{k} \rightarrow p_{k+1}$. 


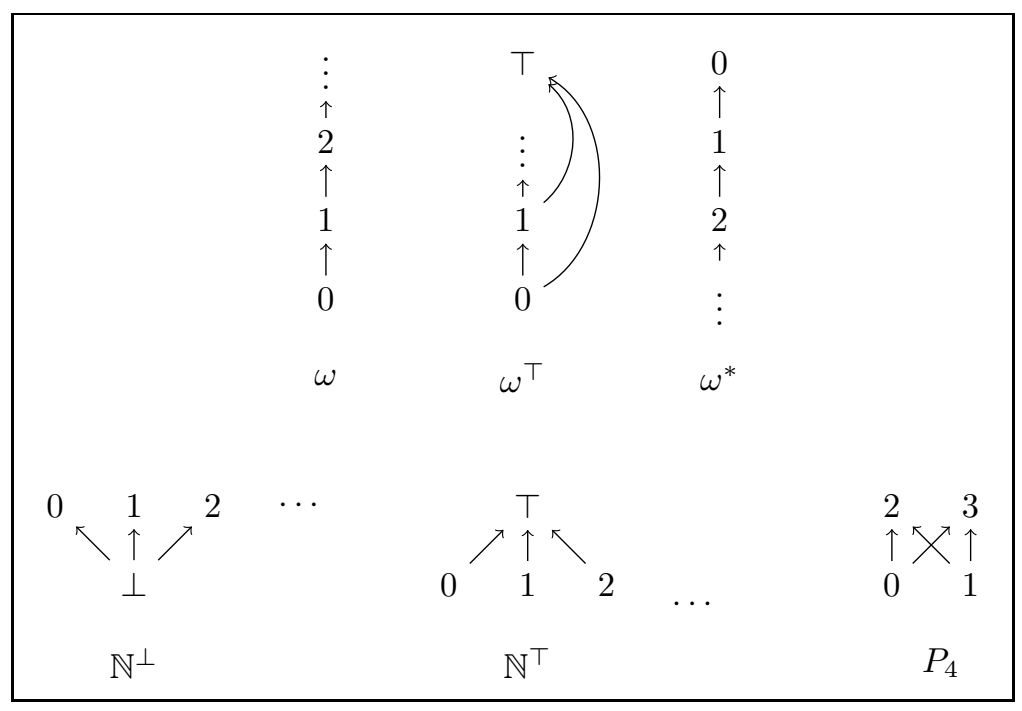

Figure 1: Samples of useful countable posets.

In Sel05], Selivanov worked with well-founded trees in order to construct subsets of $\mathcal{P} \omega$. We will generalize this construction to a larger class of posets that we call shrubs and that share some of the properties of well-founded trees. For this purpose, we make use of the classical notion of bounded completeness that occurs in domain theory.

Definition 13. A subset $S \subseteq P$ of a poset is bounded if there exists an element $p^{\prime} \in P$ - called an upper bound - such that, for any $p \in S$, we have $p \leq_{p} p^{\prime}$. If the set of all upper bounds of $S \subseteq P$ has a unique $\leq_{p}$-minimal element-i.e., if there exists an upper bound $s_{S} \in P$ of $S$ such that, for any other upper bound $p^{\prime} \in P$ of $S$, we have $s_{S} \leq_{p} p^{\prime}$ - then $s_{S}$ is called the supremum of $S$ in $P$.

$A$ poset $P$ is bounded complete if any bounded $S \subseteq P$ admits a-necessarily unique - supremum.

Notice that $P_{4}$ is a typical example of a poset which is not bounded complete, while all the other examples of Figure 1, as well as $\left(\mathcal{P}_{<\omega}(\omega), \subseteq\right)$ and $(\mathcal{P} \omega, \subseteq)$ are examples of bounded complete posets.

Definition 14. The class of all shrubs $\mathbb{P}_{\mathrm{shr}} \subseteq \mathbb{P}$ is the class of all countable posets $P \in \mathbb{P}$ that satisfy:

1. $\omega \stackrel{1-1}{\stackrel{h .}{\longrightarrow}} P$,

2. for all $p \in P, \operatorname{Card}(\operatorname{Pred}(p))<\aleph_{0}$,

3. there exists $a \leq_{p}$-minimal element $\perp$,

4. $P$ is bounded complete.

Well-founded trees and $\mathbb{N}^{\perp}$ are typical examples of shrubs. More involved ones will be constructed in the proof of Theorem 36 (Figure 5) and of Theorem 40 (Figure 6). To the contrary, $\omega, \omega^{\top}, \omega^{*}, \mathbb{N}^{\top}$, and $P_{4}$ are typical examples of posets that are not shrubs.

In the next proposition, we give alternative characterizations to the second item of the previous definition. In particular, we show that the posets we just 
defined can be embedded into $\mathcal{P}_{<\omega}(\omega)$. We also give an alternative characterization of this second item that exclusively depends on morphisms between posets.

Proposition 15. If $P \in \mathbb{P}$, then the following are equivalent:

1. for all $p \in P, \operatorname{Card}(\operatorname{Pred}(p))<\aleph_{0}$,

2. $P \stackrel{1-1 h}{\longrightarrow} \mathcal{P}<\omega(\omega)$,

3. $\left(\omega^{\top} \stackrel{1-1 / \mu}{\longrightarrow} P\right),\left(\omega^{*} \stackrel{1-1 / h .}{\longrightarrow} P\right)$ and $\left(\mathbb{N}^{\top} \stackrel{1-1 / h .}{\longrightarrow} P\right)$.

Proof.

(1. $\Rightarrow$ 2.): We consider $P \in \omega \cup\{\omega\}$ and define a function:

$$
\begin{aligned}
e: P & \rightarrow \mathcal{P}_{<\omega}(\omega) \\
k & \mapsto\left\{n \mid n \leq_{p} k\right\} .
\end{aligned}
$$

If $k \leq_{p} l$, then by transitivity of $\leq_{p}$, we get $e(k) \subseteq e(l)$. If $k \neq l$, we consider the two cases $k<_{p} l$ and $k \perp_{p} l$ (the third case $l<_{p} k$ is the same as the case $\left.k<_{p} l\right)$. In both cases, $l \in e(l) \backslash e(k)$. Therefore, we obtain that $e$ is an injective homomorphism that witnesses $P \stackrel{1-1 h .}{\longrightarrow} \mathcal{P}_{<\omega}(\omega)$.

(2. $\Rightarrow 3$.): If $\varphi: Q \stackrel{1-1 h}{\longrightarrow} P$, then for all $q \in Q$, the injectivity of $\varphi$ implies Card $(\operatorname{Pred}(q)) \leq \operatorname{Card}(\operatorname{Pred}(\varphi(q)))$. Towards a contradiction, we assume that $\left(\omega^{\top} \stackrel{1-1 h}{\longrightarrow} P\right) \vee\left(\omega^{*} \stackrel{1-1 h}{\longrightarrow} P\right) \vee\left(\mathbb{N}^{\top} \stackrel{1-1 h}{\longrightarrow} P\right)$ holds. We get a contradiction for each one of these situations:

1. if $\omega^{\top} \stackrel{1-1 h}{\longrightarrow} P$, then $\operatorname{Card}(\operatorname{Pred}(\varphi(\top)))=\aleph_{0}$,

2. if $\omega^{*} \stackrel{1-1 h}{\longrightarrow} P$, then Card $(\operatorname{Pred}(\varphi(0)))=\aleph_{0}$,

3. if $\mathbb{N}^{\top} \stackrel{1-1 h}{\longrightarrow} P$, then Card $(\operatorname{Pred}(\varphi(\top)))=\aleph_{0}$.

However, there exists no $F \in \mathcal{P}_{<\omega}(\omega)$ such that $\operatorname{Card}(\operatorname{Pred}(F))=\aleph_{0}$.

(3. $\Rightarrow$ 1.): Towards a contradiction, we pick $p \in P$ such that $\operatorname{Card}(\operatorname{Pred}(p))=$ $\aleph_{0}$. We consider three different cases.

(a) Suppose there exists $q_{0}<_{p} p$ such that there exists no immediate predecessor $p^{\prime}$ of $p$ satisfying $q_{0} \leq_{p} p^{\prime}$. Hence, there exists $q_{1}<_{p} p$ such that $q_{0}<_{p} q_{1}$. We continue the process to construct a sequence $\left(q_{n}\right)_{n \in \omega}$ witnessing $\omega^{\top} \stackrel{1-1 h}{\longrightarrow} P$ via the mapping: $\top \mapsto p$, and $n \mapsto q_{n}$ for any $n \in \omega$.

(b) Suppose there exist infinitely many immediate predecessors $\left(q_{n}\right)_{n \in \omega}$ of $p \in P$, then the mapping: $\top \mapsto p$, and $n \mapsto q_{n}$ for any $n \in \omega$, witnesses $\mathbb{N}^{\top} \stackrel{1-1 h .}{\longrightarrow} P$.

(c) Suppose that we are not in the situations (a) and (b); then, by the pigeonhole principle, there exists $q_{0}$ an immediate predecessor of $p$ such that $\operatorname{Card}\left(\operatorname{Pred}\left(q_{0}\right)\right)=\aleph_{0}$. If we replace $p$ with $q_{0}$ and start the proof again, either we get a contradiction from (a) or (b), or we exhibit $q_{1}$ an immediate predecessor of $q_{0}$ such that $\operatorname{Card}\left(\operatorname{Pred}\left(q_{1}\right)\right)=$ $\aleph_{0}$. By an infinite iteration of this process, we obtain a sequence $\left(q_{n}\right)_{n \in \omega}$ witnessing $\omega^{*} \stackrel{1-1 h}{\longrightarrow} P$ via the mapping: $0 \mapsto p$, and $n \mapsto q_{n-1}$ for any $n \in \omega^{+}$. 
In the next section, we will associate a subset $\mathcal{A}_{\mathrm{P}}$ of $\mathcal{P} \omega$ to some countable 2-colored posets $\mathrm{P}$, where the color 1 will correspond to elements inside $\mathcal{A}_{\mathrm{P}}$.

In Figure 2, we give a name to some specific 2-colored posets: the nodes of the form • and $\circ$ correspond to color 1 and color 0 , respectively.

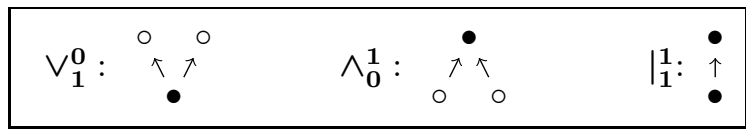

Figure 2: Samples of useful 2-colored countable posets.

The next definition introduces the class of embeddable posets. We will later associate a subset of $\mathcal{P} \omega$ to each such 2-colored poset.

Definition 16. The class of embeddable posets $\mathbb{P}_{\mathrm{emb}}$ is the class of countable 2-colored posets $\mathrm{P}=\left(P, \leq_{p}, \mathrm{c}_{p}\right)$ such that $\left(P, \leq_{p}\right) \in \mathbb{P}_{\text {shr }}$ and whose coloring satisfies:

1. $\mathrm{c}_{p}(\perp)=0$,

2. for all $k \in P \leq_{p}$-maximal, $\mathrm{c}_{p}(k)=1$,

3. $\left(\vee_{\mathbf{1}}^{\mathbf{0}} \dashv_{c} \mathrm{P}\right),\left(\wedge_{\mathbf{0}}^{\mathbf{1}} \dashv_{c} \mathrm{P}\right)$ and $\left(\left.\right|_{\mathbf{1}} ^{\mathbf{1}} \dashv_{c} \mathrm{P}\right)$.

If $\mathrm{P}$ is an embeddable poset, then the nodes of color 1 are isolated. Indeed, if $\mathrm{P} \in \mathbb{P}_{\mathrm{emb}}, p \in P$ and $\mathrm{c}_{p}(p)=1$, then $p$ has a unique immediate predecessor; and $p$ has at most one immediate successor 10 , depending on whether $p$ is $\leq_{p^{-}}$ maximal or not. Moreover, if they exist, they both have color 0 . Thus, we introduce the following notations.

Notation 17. For $\mathrm{P} \in \mathbb{P}_{\mathrm{emb}}, p \in P$ and $\mathrm{c}_{p}(p)=1$, we denote by $p^{-}$its unique immediate predecessor; and, if it exists, by $p^{+}$its unique immediate successor. We have $\mathrm{c}_{p}\left(p^{-}\right)=\mathrm{c}_{p}\left(p^{+}\right)=0$.

This means that the direct neighborhood - composed of all immediate predecessors and all immediate successors - of every node of color 1 is of one of the following form, depending on whether it is $\leq_{p}$-maximal or not:

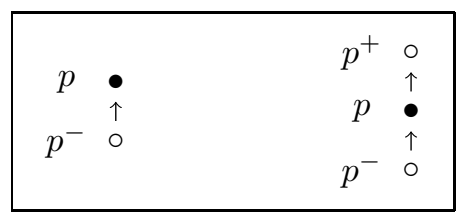

Figure 3: The two possible direct neighborhoods of any $p \in P$, where $\mathrm{P} \in \mathbb{P}_{\mathrm{emb}}$ and $c_{p}(p)=1$. The first case occurs when $p$ is $\leq_{p}$-maximal, and the second one when $p$ is not.

\footnotetext{
${ }^{10}$ If $\mathrm{P}$ is an embeddable poset, $p \in P$ is an immediate successor of $p^{\prime} \in P$ if $p^{\prime} \in \operatorname{Pred}_{\text {im }}(p)$.
} 


\section{An order-embedding into the Wadge order}

In this section, we associate a subset $\mathcal{A}_{\mathrm{P}} \in \boldsymbol{\Delta}_{2}^{0}(\mathcal{P} \omega)$ to each countable 2-colored poset $\mathrm{P}$ which is embeddable, and show that this association is such that, for any $\mathrm{P}, \mathrm{Q} \in \mathbb{P}_{\mathrm{emb}}, \mathrm{P} \preccurlyeq_{c} \mathrm{Q}$ if and only if $\mathcal{A}_{\mathrm{P}} \leq_{w} \mathcal{A}_{\mathrm{Q}}$ (Lemma 22). As a consequence, we get our main result that there exists an order-embedding $\left(\mathbb{P}_{\mathrm{emb}}, \preccurlyeq_{c}\right) / \equiv_{c} \rightarrow$ $\left(\boldsymbol{\Delta}_{2}^{0}(\mathcal{P} \omega), \leq_{w}\right) / \equiv_{w}($ Theorem 26$)$.

We first need to label the elements of any embeddable poset.

Definition 18. Let $\mathrm{P} \in \mathbb{P}_{\mathrm{emb}}$ so that $P \in \omega \cup\{\omega\}$ has $a \leq_{p}$-minimal element $m=\perp$ for some $m \in \omega$. The labeling $l_{p}$ on $P$ is defined by:

$$
\begin{aligned}
l_{p}: P & \rightarrow \mathcal{P}_{<\omega}(\omega) \\
& \mapsto \mapsto \emptyset, \\
n & \mapsto \bigcup_{k \leq_{p} n}\{k\} .
\end{aligned}
$$

We notice that $l_{p}$ is injective. Therefore, for every $F \in \mathcal{P}_{<\omega}(\omega)$ in the range of $l_{p}, l_{p}^{-1}(F)$ is well-defined.

We then associate a subset of the Scott domain to any embeddable poset through the labeling given by Definition 18.

Definition 19. Let $\mathrm{P} \in \mathbb{P}_{\mathrm{emb}}$, we define the subset $\mathcal{A}_{\mathrm{P}} \subseteq \mathcal{P} \omega$ as:

$$
\begin{aligned}
\mathcal{A}_{\mathrm{P}} & =l_{p}\left[\mathrm{c}_{p}^{-1}[\{1\}]\right] \\
& =\left\{x \subseteq \omega \mid \exists p \in P\left(\mathrm{c}_{p}(p)=1 \wedge l_{p}(p)=x\right)\right\} .
\end{aligned}
$$

We also denote by $\mathcal{C}\left(\mathcal{A}_{\mathrm{P}}\right)$ the set of all finite sets of integers contained in the labeling of an element of $P$ :

$$
\mathcal{C}\left(\mathcal{A}_{\mathrm{P}}\right)=\left\{F \subseteq \omega \mid \exists p \in P F \subseteq l_{p}(p)\right\}
$$

The next lemma gathers two crucial observations that arise from the construction given by Definition 19.

Lemma 20. Let $\mathrm{P} \in \mathbb{P}_{\mathrm{emb}}$ and $F \in \mathcal{P}_{<\omega}(\omega)$.

1. If $F \in \mathcal{C}\left(\mathcal{A}_{\mathrm{P}}\right)$, then $\left\{p \in P \mid l_{p}(p) \subseteq F\right\}$ has an upper bound in $\mathrm{P}$.

By Definition 14, it has a unique supremum denoted by $s_{F} \in P$.

2. $F \in \mathcal{A}_{\mathrm{P}} \Leftrightarrow\left(\mathrm{c}_{p}\left(s_{F}\right)=1 \wedge l_{p}\left(s_{F}\right)=F\right)$.

Proof. 1. Since $F \in \mathcal{C}\left(\mathcal{A}_{\mathrm{P}}\right)=\left\{F \subseteq \omega \mid \exists p \in P F \subseteq l_{p}(p)\right\}$, there exists $p_{0} \in P$ such that $F \subseteq l_{p}\left(p_{0}\right)$. Thus, $p_{0} \in P$ is an upper bound of $\{p \in P \mid$ $\left.l_{p}(p) \subseteq F\right\}$.

2. Assume first that $F \in \mathcal{A}_{\mathrm{P}} \subseteq \mathcal{C}\left(\mathcal{A}_{\mathrm{P}}\right)$. Then, there exists $p_{0} \in P$ such that $\mathrm{c}_{p}\left(p_{0}\right)=1$ and $l_{p}\left(p_{0}\right)=F$. It implies that $p_{0} \leq_{p} s_{F}$. Since $s_{F}$ is the supremum of $\left\{p \in P \mid l_{p}(p) \subseteq F\right\}$ and $s_{F}$ has a unique immediate predecessor, we have $s_{F} \in\left\{p \in P \mid l_{p}(p) \subseteq F\right\}$. Thus $F=l_{p}\left(p_{0}\right) \subseteq l_{p}\left(s_{F}\right) \subseteq F$. By injectivity of $l_{p}$, we obtain $s_{F}=p_{0}$ and $c_{p}\left(s_{F}\right)=1$.

Conversely, from the very definition of $\mathcal{A}_{\mathrm{P}}$, we have $\mathrm{c}_{p}\left(s_{F}\right)=1$ and $l_{p}\left(s_{F}\right)=F$, which implies that $F \in \mathcal{A}_{\mathrm{P}}$. 
The rest of this section consists in proving that the correspondence $\mathrm{P} \mapsto \mathcal{A}_{\mathrm{P}}$ satisfies that $\mathcal{A}_{\mathrm{P}} \in \boldsymbol{\Delta}_{2}^{0}(\mathcal{P} \omega)$ and for any $\mathrm{P}, \mathrm{Q} \in \mathbb{P}_{\mathrm{emb}}, \mathrm{P} \preccurlyeq_{c} \mathrm{Q}$ if and only if $\mathcal{A}_{\mathrm{P}} \leq_{w} \mathcal{A}_{\mathrm{Q}}$. For this, we need a result which claims that a continuous mapping from $\mathcal{P} \omega$ to itself is completely determined by its behavior on $\mathcal{P}_{<\omega}(\omega)$.

Lemma 21 (Exercice 5.1.62 in [GL13]). Given any $\subseteq$-increasing mapping $f$ : $\mathcal{P}_{<\omega}(\omega) \rightarrow \mathcal{P} \omega$, there exists a unique continuous extension of $f$ to the whole Scott domain. This extension is given by

$$
\begin{aligned}
\hat{f}: \mathcal{P} \omega & \rightarrow \mathcal{P} \omega \\
x & \mapsto \bigcup_{n \in \omega} f(x \cap n) .
\end{aligned}
$$

Proof.

Existence: It suffices to prove that $\hat{f}$ is continuous. Observe that, for all $x \in \mathcal{P} \omega$, the sequence $(f(x \cap n))_{n \in \omega}$ is $\subseteq$-increasing. Let $F \in \mathcal{P}_{<\omega}(\omega)$ such that $\mathcal{O}_{F}$ is a basic open set. If $x \in \hat{f}^{-1}\left[\mathcal{O}_{F}\right]$, then $F \subseteq \hat{f}(x)$. Since $F$ is finite, there exists $n_{0} \in \omega$ such that $F \subseteq f\left(x \cap n_{0}\right)$. We obtain

$$
x \in \mathcal{O}_{x \cap n_{0}} \subseteq \hat{f}^{-1}\left[\mathcal{O}_{F}\right]
$$

which shows that $\hat{f}$ is continuous.

Uniqueness: Observe that a continuous function has to be $\subseteq$-increasing on the whole domain. This follows from the $\subseteq$-upward closure of the open subsets of $\mathcal{P} \omega$. Let $g: \mathcal{P} \omega \rightarrow \mathcal{P} \omega$ be any continuous extension of $f$. Given any $x \in \mathcal{P} \omega$, consider $k \in g(x)$. By continuity, there exists $F \in \mathcal{P}_{<\omega}(\omega)$ such that $x \in \mathcal{O}_{F} \subseteq g^{-1}\left[\mathcal{O}_{\{k\}}\right]$. Because $F$ is finite, there exists $n_{0} \in \omega$ such that $x \cap n_{0} \in \mathcal{O}_{F}$. Thus, $k \in g\left(x \cap n_{0}\right)=f\left(x \cap n_{0}\right) \subseteq \hat{f}(x)$. The exact same reasoning works if $g$ and $\hat{f}$ are swapped. Hence, we conclude that for all $x \in \mathcal{P} \omega$, we have $\hat{f}(x)=g(x)$.

We are now ready for our main proof.

Lemma 22. The following mapping

$$
\begin{aligned}
H:\left(\mathbb{P}_{\mathrm{emb}}, \preccurlyeq_{c}\right) & \rightarrow\left(\boldsymbol{\Delta}_{2}^{0}(\mathcal{P} \omega), \leq_{w}\right) \\
\mathrm{P} & \mapsto \mathcal{A}_{\mathrm{P}}
\end{aligned}
$$

satisfies that for any $\mathrm{P}, \mathrm{Q} \in \mathbb{P}_{\mathrm{emb}}$, we have

$$
\mathrm{P} \preccurlyeq{ }_{c} \mathrm{Q} \text { if and only if } \mathcal{A}_{\mathrm{P}} \leq_{w} \mathcal{A}_{\mathrm{Q}} \text {. }
$$

Proof. The proof is divided into the three Claims 23, 24 and 25. The first two claims show that $H$ is well-defined and order-preserving, while the third one completes the proof.

Claim 23. If $\mathrm{P} \in \mathbb{P}_{\mathrm{emb}}$, then $\mathcal{A}_{\mathrm{P}} \in \boldsymbol{\Delta}_{2}^{0}(\mathcal{P} \omega)$. 
Proof of the claim. We show that $\mathcal{A}_{\mathrm{P}}$ is both approximable and co-approximable. $\mathcal{A}_{\mathrm{P}}$ is approximable because $\mathcal{A}_{\mathrm{P}} \subseteq \mathcal{P}_{<\omega}(\omega)$. For co-approximability, we proceed by contradiction and suppose that $\mathcal{A}_{\mathrm{P}}$ is not co-approximable for some $x \in$ $\mathcal{P} \omega \backslash \mathcal{A}_{\mathrm{P}}$ infinite. So, we fix $F_{0} \in[\emptyset, x] \cap \mathcal{A}_{\mathrm{P}}$ and set $p_{0}=l_{p}^{-1}\left(F_{0}\right)$. Assume $F_{n}$ and $p_{n}$ are already constructed. Since $\mathcal{A}_{\mathrm{P}}$ is not co-approximable, there exists $F_{n+1} \in\left(\left[F_{n}, x\right] \backslash\left\{F_{n}\right\}\right) \cap \mathcal{A}_{\mathrm{P}}$. We set $p_{n+1}=l_{p}^{-1}\left(F_{n+1}\right)$. It follows that the function

$$
\begin{aligned}
\varphi: \omega & \rightarrow P \\
n & \mapsto p_{n}
\end{aligned}
$$

witnesses $\omega \stackrel{1-1 h}{\longrightarrow} P$, a contradiction.

Claim

Claim 24. If $\mathrm{P}, \mathrm{Q} \in \mathbb{P}_{\mathrm{emb}}$ and $\mathrm{P} \preccurlyeq \preccurlyeq_{c} \mathrm{Q}$, then $\mathcal{A}_{\mathrm{P}} \leq_{w} \mathcal{A}_{\mathrm{Q}}$.

Proof of the claim. Suppose that $\mathrm{P} \preccurlyeq c \mathrm{Q}$ is witnessed by $\varphi: P \rightarrow Q$. Consider the function:

$$
\begin{aligned}
f_{\varphi}: \mathcal{P}_{<\omega}(\omega) \rightarrow \mathcal{P} \omega & \begin{cases}l_{q}\left(\varphi\left(s_{F}\right)\right) & \text { if } F \in \mathcal{C}\left(\mathcal{A}_{\mathrm{P}}\right) \wedge c_{p}\left(s_{F}\right)=0, \\
l_{q}\left(\varphi\left(s_{F}\right)\right) & \text { if } F \in \mathcal{C}\left(\mathcal{A}_{\mathrm{P}}\right) \wedge c_{p}\left(s_{F}\right)=1 \wedge F=l_{p}\left(s_{F}\right), \\
l_{q}\left(\varphi\left(s_{F}^{-}\right)\right) & \text {if } F \in \mathcal{C}\left(\mathcal{A}_{\mathrm{P}}\right) \wedge c_{p}\left(s_{F}\right)=1 \wedge F \subsetneq l_{p}\left(s_{F}\right), \\
l_{q}\left(\varphi\left(s_{F}^{+}\right)\right) & \text {if } F \in \mathcal{C}\left(\mathcal{A}_{\mathrm{P}}\right) \wedge c_{p}\left(s_{F}\right)=1 \wedge F \nsubseteq l_{p}\left(s_{F}\right), \\
\omega & \text { otherwise, }\end{cases}
\end{aligned}
$$

where $s_{F}$ is defined as in Lemma 20, $s_{F}^{-}$and $s_{F}^{-}$are defined as in Notation 17, and $s_{F}^{+}$is replaced by $\omega$ whenever $s_{F}$ is a maximal element in $\left(P, \leq_{p}\right)$.

We show that the function $\hat{f}_{\varphi}$ given by Lemma 21 satisfies $\hat{f}_{\varphi}^{-1}\left[\mathcal{A}_{Q}\right]=\mathcal{A}_{\mathrm{P}}$. First, for $\hat{f}_{\varphi}$ to exist, we need $f_{\varphi}$ to be increasing. Let $F, G \in \mathcal{P}_{<\omega}(\omega)$ be such that $F \subseteq G$. We have several cases to check:

1. if $G \notin \mathcal{C}\left(\mathcal{A}_{\mathrm{P}}\right)$, then $f_{\varphi}(F) \subseteq f_{\varphi}(G)=\omega$.

Since $G \in \mathcal{C}\left(\mathcal{A}_{\mathrm{P}}\right)$ implies $F \in \mathcal{C}\left(\mathcal{A}_{\mathrm{P}}\right)$, we now suppose $F, G \in \mathcal{C}\left(\mathcal{A}_{\mathrm{P}}\right)$ and thus $s_{F} \leq_{p} s_{G}$.

2. if $\mathrm{c}_{p}\left(s_{F}\right)=\mathrm{c}_{p}\left(s_{G}\right)=0$, then $f_{\varphi}(F)=l_{q}\left(\varphi\left(s_{F}\right)\right) \subseteq l_{q}\left(\varphi\left(s_{G}\right)\right)=f_{\varphi}(G)$,

3. if $\mathrm{c}_{p}\left(s_{F}\right)=0$ and $\mathrm{c}_{p}\left(s_{G}\right)=1$, then $f_{\varphi}(F)=l_{q}\left(\varphi\left(s_{F}\right)\right) \subseteq l_{q}\left(\varphi\left(s_{G}^{-}\right)\right) \subseteq$ $f_{\varphi}(G)$

4. if $\mathrm{c}_{p}\left(s_{F}\right)=1$ and $\mathrm{c}_{p}\left(s_{G}\right)=0$, then $f_{\varphi}(F) \subseteq l_{q}\left(\varphi\left(s_{F}^{+}\right)\right) \subseteq l_{q}\left(\varphi\left(s_{G}\right)\right)=$ $f_{\varphi}(G)$,

5. if $\mathrm{c}_{p}\left(s_{F}\right)=\mathrm{c}_{p}\left(s_{G}\right)=1$ and $s_{F} \neq s_{G}$, then there exists $p \in P$ such that $s_{F}<_{p} p<_{p} s_{G}$ holds, because there exist no two consecutive nodes colored by 1 . Therefore $f_{\varphi}(F) \subseteq l_{q}\left(\varphi\left(s_{F}^{+}\right)\right) \subseteq l_{q}\left(\varphi\left(s_{G}^{-}\right)\right)=f_{\varphi}(G)$.

It only remains to consider the cases where $\mathrm{c}_{p}\left(s_{F}\right)=\mathrm{c}_{p}\left(s_{G}\right)=1$, and $s_{F}=s_{G}$ :

6. if $F, G \in \mathcal{A}_{\mathrm{P}}$, then $f_{\varphi}(F)=l_{q}\left(\varphi\left(s_{F}\right)\right)=l_{q}\left(\varphi\left(s_{G}\right)\right)=f_{\varphi}(G)$,

7. if $F \in \mathcal{A}_{\mathrm{P}}$ and $G \notin \mathcal{A}_{\mathrm{P}}$, then $f_{\varphi}(F)=l_{q}\left(\varphi\left(s_{F}\right)\right) \subseteq l_{q}\left(\varphi\left(s_{F}^{+}\right)\right)=f_{\varphi}(G)$,

8. if $F \notin \mathcal{A}_{\mathrm{P}}$ and $G \in \mathcal{A}_{\mathrm{P}}$, then $f_{\varphi}(F)=l_{q}\left(\varphi\left(s_{F}^{-}\right)\right) \subseteq l_{q}\left(\varphi\left(s_{F}\right)\right)=f_{\varphi}(G)$, 
9. if $F, G \notin \mathcal{A}_{\mathrm{P}}$ and $F \subsetneq l_{p}\left(s_{F}\right)$, then $f_{\varphi}(F)=l_{q}\left(\varphi\left(s_{F}^{-}\right)\right) \subseteq f_{\varphi}(G)$,

10. if $F, G \notin \mathcal{A}_{\mathrm{P}}$ and $F \nsubseteq l_{p}\left(s_{F}\right)$, then $f_{\varphi}(F)=l_{q}\left(\varphi\left(s_{F}^{+}\right)\right)=f_{\varphi}(G)$.

This finishes the proof that $f_{\varphi}: \mathcal{P}_{<\omega}(\omega) \rightarrow \mathcal{P} \omega$ is increasing. It follows from Lemma 21, that $f_{\varphi}$ has a continuous extension $\hat{f}_{\varphi}: \mathcal{P} \omega \rightarrow \mathcal{P} \omega$. We distinguish between three different cases to show that $\hat{f}_{\varphi}^{-1}\left[\mathcal{A}_{Q}\right]=\mathcal{A}_{\mathrm{P}}$.

$\boldsymbol{x} \in \mathcal{P}_{\boldsymbol{\omega}}(\boldsymbol{\omega})$ : because $\mathcal{A}_{\mathrm{P}} \subseteq \mathcal{P}_{<\omega}(\omega)$, we have $x \notin \mathcal{A}_{\mathrm{P}}$. Suppose, towards a contradiction, that $\hat{f}_{\varphi}(x) \in \mathcal{A}_{Q}$. Since $\mathcal{A}_{Q} \subseteq \mathcal{P}_{<\omega}(\omega)$, there exist $F \in$ $\mathcal{P}_{<\omega}(\omega)$ and $n \in \omega$, such that $\hat{f}_{\varphi}(x)=F \in \mathcal{A}_{Q}$ and $f_{\varphi}(x \cap m)=F$ both hold for all $m \geq n$. We then notice that

$$
\begin{aligned}
f_{\varphi}(G) \in \mathcal{A}_{\mathrm{Q}} & \Rightarrow G \in \mathcal{C}\left(\mathcal{A}_{\mathrm{P}}\right) \wedge \mathrm{c}_{p}\left(s_{G}\right)=1 \wedge G=l_{p}\left(s_{G}\right) \\
& \Rightarrow G \in \mathcal{A}_{\mathrm{P}} .
\end{aligned}
$$

Where the first implication comes from the definition of $f_{\varphi}$ and the second from Lemma 20. We obtain that $x \cap m \in \mathcal{A}_{\mathrm{P}}$ holds for all $m \geq n$, this implies $c_{p}\left(l_{p}^{-1}(x \cap m)\right)=1$, and since $x$ is infinite, we can extract a subsequence of $\left(l_{p}^{-1}(x \cap m)\right)_{m \in \omega}$ witnessing $\omega \stackrel{1-1 h}{\longrightarrow} P$, a contradiction.

$\boldsymbol{F} \in \mathcal{P}_{<\omega}(\omega) \backslash \mathcal{C}\left(\mathcal{A}_{\mathrm{P}}\right): F \notin \mathcal{A}_{\mathrm{P}}$ holds by the very definition of $\mathcal{C}\left(\mathcal{A}_{\mathrm{P}}\right)$. Hence, we have $\omega=f_{\varphi}(F)=\hat{f}_{\varphi}(F) \notin \mathcal{A}_{Q}$.

$\boldsymbol{F} \in \mathcal{C}\left(\mathcal{A}_{\mathbf{P}}\right):$ Suppose first that $F \in \mathcal{A}_{\mathbf{P}}$. By Lemma 20, $\hat{f}_{\varphi}(F)=l_{q}\left(\varphi\left(s_{F}\right)\right)$ is satisfied. Moreover, from $\mathrm{c}_{q}\left(\varphi\left(s_{F}\right)\right)=1$, we get $\hat{f}_{\varphi}(F) \in \mathcal{A}_{\mathbf{Q}}$.

Suppose now that $F \notin \mathcal{A}_{\mathrm{P}}$. By Lemma 20, there are three cases:

1. if $\mathrm{c}_{p}\left(s_{F}\right)=0$, then $\mathrm{c}_{q}\left(\varphi\left(s_{F}\right)\right)=0$ which implies $\hat{f}_{\varphi}(F) \notin \mathcal{A}_{Q}$,

2. if $\mathrm{c}_{p}\left(s_{F}\right)=1$ and $F \subsetneq l_{p}\left(s_{F}\right)$, then $\mathrm{c}_{q}\left(\varphi\left(s_{F}^{-}\right)\right)=0$ which implies $\hat{f}_{\varphi}(F) \notin \mathcal{A}_{Q}$,

3. if $\mathrm{c}_{p}\left(s_{F}\right)=1$ and $F \nsubseteq l_{p}\left(s_{F}\right)$, then $\mathrm{c}_{q}\left(\varphi\left(s_{F}^{+}\right)\right)=0$ which implies $\hat{f}_{\varphi}(F) \notin \mathcal{A}_{Q}$.

Claim

Claim 25. If $\mathrm{P}, \mathrm{Q} \in \mathbb{P}_{\mathrm{emb}}$ and $\mathcal{A}_{\mathrm{P}} \leq_{w} \mathcal{A}_{\mathrm{Q}}$, then $\mathrm{P} \preccurlyeq{ }_{c} \mathrm{Q}$.

Proof of the claim. We assume that $\mathcal{A}_{\mathrm{P}} \leq_{w} \mathcal{A}_{\mathrm{Q}}$ is witnessed by some continuous function $f: \mathcal{P} \omega \rightarrow \mathcal{P} \omega$. We describe a reduction which witnesses $\mathrm{P} \preccurlyeq c$ Q First, we need a few observations. Let $p \in P$. Since $\omega \stackrel{1-1 / h}{\longrightarrow} P$ and all $\leq_{p^{-}}$ maximal elements have color 1 , there exists $p^{\prime} \in P$ such that both $p \leq_{p} p^{\prime}$ and $\mathrm{c}_{p}\left(p^{\prime}\right)=1$ hold. Therefore, $f\left(l_{p}\left(p^{\prime}\right)\right) \in \mathcal{A}_{\mathbf{Q}}$. Hence, for all $p \in P$, we have $f\left(l_{p}(p)\right) \in \mathcal{C}\left(\mathcal{A}_{\mathbf{Q}}\right)$. We also define, for all $p \in P$, the set

$$
Q_{p}=\left\{q \in Q \mid l_{q}(q) \subseteq f\left(l_{p}(p)\right)\right\} .
$$

Since $f\left(l_{p}(p)\right) \in \mathcal{C}\left(\mathcal{A}_{\mathbf{Q}}\right)$ holds, Lemma 20 yields the existence of a unique supremum $t_{p}$ of $Q_{p}$ in $Q$. 
We define a mapping:

$$
\begin{aligned}
\varphi: P \rightarrow Q \\
p \mapsto \begin{cases}t_{p} & \text { if } f\left(l_{p}(p)\right) \in \mathcal{A}_{\mathrm{Q}}, \\
t_{p} & \text { if } f\left(l_{p}(p)\right) \notin \mathcal{A}_{\mathrm{Q}} \wedge \mathrm{c}_{q}\left(t_{p}\right)=0, \\
t_{p}^{-} & \text {if } f\left(l_{p}(p)\right) \notin \mathcal{A}_{\mathrm{Q}} \wedge \mathrm{c}_{q}\left(t_{p}\right)=1 \wedge l_{q}\left(t_{p}\right) \subsetneq f\left(l_{p}(p)\right), \\
t_{p}^{+} & \text {if } f\left(l_{p}(p)\right) \notin \mathcal{A}_{\mathrm{Q}} \wedge c_{q}\left(t_{p}\right)=1 \wedge l_{q}\left(t_{p}\right) \nsubseteq f\left(l_{p}(p)\right),\end{cases}
\end{aligned}
$$

where $t_{p}^{-}$and $t_{p}^{+}$are defined as in Notation 17.

For $\varphi$ to be well-defined, we need $t_{p}^{+}$not to occur whenever $t_{p}$ is a $\leq_{q^{-}}$ maximal element. So, suppose $t_{p}$ is a $\leq_{q}$-maximal element. Since $\mathrm{c}_{q}\left(t_{p}\right)=1$, then $t_{p} \in Q_{p}$ for it has a unique immediate predecessor. Thus, $l_{q}\left(t_{p}\right) \subseteq f\left(l_{p}(p)\right)$ holds, which shows that $t_{p}^{+}$does not occur in this case.

Since for every $p \in P$ we have

$$
\mathrm{c}_{p}(p)=1 \Leftrightarrow l_{p}(p) \in \mathcal{A}_{\mathrm{P}} \Leftrightarrow f\left(l_{p}(p)\right) \in \mathcal{A}_{\mathrm{Q}},
$$

it follows from the definition of $\varphi$, that for all $p \in P$ we also have $c_{p}(p)=$ $\mathrm{c}_{q}(\varphi(p))$. Therefore, it only remains to show that $\varphi$ is order-preserving. Suppose $p \leq_{p} p^{\prime}$, we get $t_{p} \leq_{q} t_{p^{\prime}}$. We proceed with cases:

1. if $\mathrm{c}_{q}\left(t_{p}\right)=\mathrm{c}_{q}\left(t_{p^{\prime}}\right)=0$, then $\varphi(p)=t_{p} \leq_{q} t_{p^{\prime}}=\varphi\left(p^{\prime}\right)$,

2. if $\mathrm{c}_{q}\left(t_{p}\right)=0$ and $\mathrm{c}_{q}\left(t_{p^{\prime}}\right)=1$, then $\varphi(p)=t_{p} \leq_{q} t_{p^{\prime}}^{-} \leq_{q} \varphi\left(p^{\prime}\right)$,

3. if $\mathrm{c}_{q}\left(t_{p}\right)=1$ and $\mathrm{c}_{q}\left(t_{p^{\prime}}\right)=0$, then $\varphi(p) \leq_{q} t_{p}^{+} \leq_{q} t_{p^{\prime}}=\varphi\left(p^{\prime}\right)$,

4. if $\mathrm{c}_{q}\left(t_{p}\right)=\mathrm{c}_{q}\left(t_{p^{\prime}}\right)=1$ and $t_{p} \neq t_{p^{\prime}}$, then there exists some $q \in Q$ that satisfies $t_{p}<_{q} q<_{q} t_{p^{\prime}}$. This finally leads to $\varphi(p) \leq_{q} t_{p}^{+} \leq_{q} t_{p^{\prime}}^{-}=\varphi\left(p^{\prime}\right)$.

It only remains to consider the cases where $\mathrm{c}_{q}\left(t_{p}\right)=\mathrm{c}_{q}\left(t_{p^{\prime}}\right)=1$, and $t_{p}=t_{p^{\prime}}$ :

5. if $\mathrm{c}_{p}(p)=\mathrm{c}_{p}\left(p^{\prime}\right)=1$, then $\varphi(p)=t_{p}=t_{p^{\prime}}=\varphi\left(p^{\prime}\right)$,

6. if $\mathrm{c}_{p}(p)=1$ and $\mathrm{c}_{p}\left(p^{\prime}\right)=0$, then $\varphi(p)=t_{p} \leq_{q} t_{p}^{+}=\varphi\left(p^{\prime}\right)$,

7. if $\mathrm{c}_{p}(p)=0$ and $\mathrm{c}_{p}\left(p^{\prime}\right)=1$, then $\varphi(p)=t_{p}^{-} \leq_{q} t_{p}=\varphi\left(p^{\prime}\right)$,

8. if $\mathrm{c}_{p}(p)=\mathrm{c}_{p}\left(p^{\prime}\right)=0$ and $l_{q}\left(t_{p}\right) \subsetneq f\left(l_{p}(p)\right)$, then $\varphi(p)=t_{p}^{-} \leq_{q} \varphi\left(p^{\prime}\right)$,

9. if $\mathrm{c}_{p}(p)=\mathrm{c}_{p}\left(p^{\prime}\right)=0$ and $l_{q}\left(t_{p}\right) \nsubseteq f\left(l_{p}(p)\right)$, then $\varphi(p)=t_{p}^{+}=\varphi\left(p^{\prime}\right)$.

This concludes the proof that $\varphi$ witnesses $\mathrm{P} \preccurlyeq c$ $\mathrm{Q}$.

Claim

So, Claim 23 proves that the mapping $H: \mathrm{P} \mapsto \mathcal{A}_{\mathrm{P}}$ is a well-defined mapping from $\left(\mathbb{P}_{\mathrm{emb}}, \preccurlyeq_{c}\right)$ to $\left(\boldsymbol{\Delta}_{2}^{0}(\mathcal{P} \omega), \leq_{w}\right)$, and we conclude from the Claims 24 and 25 that for any $\mathrm{P}, \mathrm{Q} \in \mathbb{P}_{\mathrm{emb}}, \mathrm{P} \preccurlyeq_{c} \mathrm{Q}$ if and only if $\mathcal{A}_{\mathrm{P}} \leq_{w} \mathcal{A}_{\mathrm{Q}}$.

The previous lemma almost immediately yields the main result:

Theorem 26. The following mapping is an order-embedding:

$$
\begin{aligned}
\left(\mathbb{P}_{\mathrm{emb}}, \preccurlyeq_{c}\right) / \equiv_{c} & \rightarrow\left(\boldsymbol{\Delta}_{2}^{0}(\mathcal{P} \omega), \leq_{w}\right) / \equiv_{w} \\
{[\mathrm{P}] } & \mapsto\left[\mathcal{A}_{\mathrm{P}}\right] .
\end{aligned}
$$

Proof. By Lemma 22 and the definition of the order on quotient sets, it is clear that for any $[\mathrm{P}],[\mathrm{Q}] \in\left(\mathbb{P}_{\mathrm{emb}}, \preccurlyeq_{c}\right) / \equiv_{c}$, we have $[\mathrm{P}] \preccurlyeq_{c}[\mathrm{Q}]$ if and only if $\left[\mathcal{A}_{\mathrm{P}}\right] \leq_{w}\left[\mathcal{A}_{\mathrm{Q}}\right]$. Moreover, if $\left[\mathcal{A}_{\mathrm{P}}\right]=\left[\mathcal{A}_{\mathrm{Q}}\right]$, then $\mathcal{A}_{\mathrm{P}} \equiv_{w} \mathcal{A}_{\mathrm{Q}}$, and by Lemma 22, we have $\mathrm{P} \equiv{ }_{c} \mathrm{Q}$, hence $[\mathrm{P}]=[\mathrm{Q}]$. Thus, the mapping $[\mathrm{P}] \mapsto\left[\mathcal{A}_{\mathrm{P}}\right]$ is an order-embedding. 


\section{A reduction game on $\mathbb{P}$}

This section introduces a game characterization of reductions on 2-colored posets. This characterization and the order-embedding given in Theorem 26 are the essential tools that we need in order to study the Wadge order on the Scott domain.

This game comes as a standard two-player infinite game where the players choose elements of $\mathbb{P}$.

Definition 27. Let $\mathrm{P}, \mathrm{Q} \in \mathbb{P}$. The game $G_{\mathbb{P}}(\mathrm{P}, \mathrm{Q})$ is defined as a two-player (I and II) game played on $\omega$ rounds. Each round $n \in \omega$ is played as follows: first I picks an element $p_{n} \in P$ and then II picks an element $q_{n} \in Q$. We further require that there exists $n_{0} \in \omega$ such that, for all $n \geq n_{0}, p_{n}=p_{n_{0}}$.

We say that II wins the game if and only if the two following conditions are satisfied:

1. $p_{n} \leq_{p} p_{m} \rightarrow q_{n} \leq_{q} q_{m}$ holds for all $n, m \in \omega$,

2. $\mathrm{c}_{p}\left(p_{n}\right)=\mathrm{c}_{q}\left(q_{n}\right)$ for all $n \in \omega$.

Schematically, the game goes as follows:

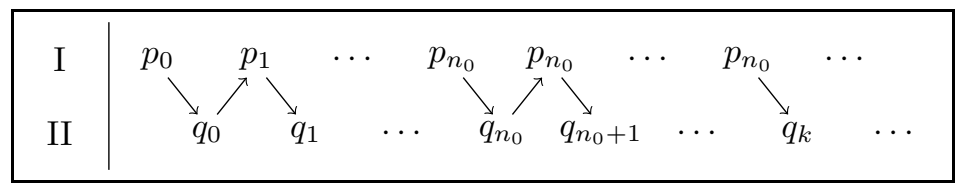

Figure 4: The game $G_{\mathbb{P}}(P, Q)$ for $\mathrm{P}, \mathrm{Q} \in \mathbb{P}$.

$A$ run of the game is a sequence $\left(p_{0}, q_{0}, p_{1}, q_{1}, \ldots\right) \in(P \cup Q)^{\omega}$.

In plain English, player I moves inside the 2-colored poset $\mathrm{P}$, whereas player II moves inside the 2-colored poset Q. The goal of II is to reproduce (order-wise and color-wise) in $Q$ the run that I is producing in P. Notice that the condition of playing ultimately constant for player I is equivalent to requiring that the game stops after finitely many rounds.

Related to this game, we introduce the notion of an ultrapositional strategy as a strengthening of the usual notion of a strategy.

Definition 28. Let $\mathrm{P}, \mathrm{Q} \in \mathbb{P}$. An ultrapositional strategy for player II in the game $G_{\mathbb{P}}(\mathrm{P}, \mathrm{Q})$ is a function $\tau: P \rightarrow Q$.

Contrary to the usual strategies that rely on the history of the opponent's run, ultrapositional strategies only take into account the last move of the opponent. An ultrapositional strategy is winning if it ensures a win whatever the opponent does.

Ultrapositional strategies characterize the reductions inside $\mathbb{P}$ as shown by the next proposition.

Proposition 29. Let $\mathrm{P}, \mathrm{Q} \in \mathbb{P}$.

$$
\mathrm{P} \preccurlyeq_{c} \mathrm{Q} \Longleftrightarrow \mathrm{II} \text { has an ultrapositional winning strategy in } G_{\mathbb{P}}(\mathrm{P}, \mathrm{Q}) \text {. }
$$

Proof. First, suppose that $\mathrm{P} \preccurlyeq_{c} \mathrm{Q}$ holds and is witnessed by $\varphi: P \rightarrow Q$. Observe that $\varphi$ is also an ultrapositional strategy. From the very definition of 
a homomorphism between 2-colored posets, it respects the two conditions to be winning for II in $G_{\mathbb{P}}(\mathrm{P}, \mathrm{Q})$.

Conversely, an ultrapositional winning strategy for II in $G_{\mathbb{P}}(\mathrm{P}, \mathrm{Q})$ is a homomorphism $\varphi: P \rightarrow Q$ for it respects the two winning conditions.

We obtain a reduction between 2-colored posets and their subposets that are closed under the predecessor relation.

Definition 30. Let $\left(Q, \leq_{q}\right)$ be a poset. A subposet $\left(P, \leq_{p}\right)$ is an ideal of $\left(Q, \leq_{q}\right)$ if, for all $p \in P$, we have $\left\{q \in Q: q \leq_{q} p\right\} \subseteq P$.

Proposition 31. Let $\mathrm{P}, \mathrm{Q} \in \mathbb{P}$.

$$
\text { If } \mathrm{P} \text { is an ideal of } \mathrm{Q} \text {, then } \mathrm{P} \preccurlyeq c \mathrm{Q} \text {. }
$$

Proof. The inclusion $i: P \rightarrow Q, p \mapsto p$ witnessing that $\left(P, \leq_{p}\right)$ is an ideal of $\left(Q, \leq_{q}\right)$ is an ultrapositional winning strategy for II in $G_{\mathbb{P}}(\mathrm{P}, \mathrm{Q})$.

\subsection{On the reduction game on $\mathbb{P}_{\text {fin }}$}

In order to simplify some later proofs, we conclude this section with some necessary conditions for an ultrapositional strategy to be winning in a subclass of the embeddable posets.

Definition 32. A finite branching poset is an embeddable poset $\mathrm{P} \in \mathbb{P}_{\mathrm{emb}}$ such that every element $p \in P$ which is not $\leq_{p}$-minimal has finitely many successors, i.e., for all $p \in P$, if $p \neq \perp$, then:

$$
\operatorname{Card}(\operatorname{Succ}(p))=\operatorname{Card}\left(\left\{p^{\prime} \in P \mid p \leq_{p} p^{\prime}\right\}\right)<\aleph_{0} .
$$

The class of all finite branching posets is denoted by $\mathbb{P}_{\text {fin }}$.

It turns out that the image of a finitely branching poset via the orderembedding of Theorem 26 must be topologically reasonably simple, for we have:

Proposition 33. If $\mathrm{P} \in \mathbb{P}_{\text {fin }}$, then $\mathcal{A}_{\mathrm{P}} \in D_{\omega}\left(\boldsymbol{\Sigma}_{1}^{0}\right)(\mathcal{P} \omega)$.

Proof. We use the characterization of Corollary 12, Since $\mathrm{P} \in \mathbb{P}_{\text {emb holds, }}$ Lemma 22 implies that $\mathcal{A}_{\mathrm{P}} \in \boldsymbol{\Delta}_{2}^{0}(\mathcal{P} \omega)$ holds as well. Towards a contradiction, assume that $\mathcal{A}_{\mathrm{P}}$ admits a 1 -alternating tree of rank $\omega$, namely:

$$
f: T_{\omega} \rightarrow \mathcal{P}_{<\omega}(\omega) .
$$

This implies that, for every $k \in \omega$, there exists a strictly $\subseteq$-increasing sequence $\left(F_{m}^{k}\right)_{m<k}$ such that $F_{0}^{k}=f(\varnothing)$ and $F_{m}^{k} \in \mathcal{A}_{\mathrm{P}}$ both hold for all $m<k$. Thus, the sequence $\left(l_{p}^{-1}\left(F_{m}^{k}\right)\right)_{l<k}$ is a strictly $\leq_{p}$-increasing sequence of size $k$ that satisfies

$$
c_{p}\left(l_{p}^{-1}\left(F_{0}^{k}\right)\right)=c_{p}\left(l_{p}^{-1}(f(\varnothing))\right)=1,
$$

for every $k \in \omega$. Therefore, we obtain

$$
\operatorname{Card}\left(\operatorname{Succ}\left(l_{p}^{-1}(f(\varnothing))\right)\right)=\aleph_{0} .
$$

By definition of a finite branching poset, this implies $l_{p}^{-1}(f(\varnothing))=\perp$, a contradiction for $\mathrm{c}_{p}(\perp)=0$. 
As a corollary, we obtain a somehow more detailed picture of Theorem 26 .

Corollary 34. The following mapping is an order-embedding:

$$
\begin{aligned}
H:\left(\mathbb{P}_{\text {fin }}, \preccurlyeq_{c}\right) / \equiv_{c} & \rightarrow\left(D_{\omega}\left(\Sigma_{1}^{0}\right)(\mathcal{P} \omega), \leq_{w}\right) / \equiv_{w} \\
{[\mathrm{P}] } & \mapsto\left[\mathcal{A}_{\mathrm{P}}\right] .
\end{aligned}
$$

Now, we introduce some notations to talk about the game-theoretical strength of a given node in a finite branching poset.

Let us fix $\mathrm{P} \in \mathbb{P}_{\text {fin }}$ and $p \in P$. If it exists, let $k_{p} \in \omega$ be the length of the largest strictly $\leq_{p}$-increasing sequence $\left(s_{n}\right)_{n<k_{p}}$ that satisfies $s_{0}=p$ and $\left(\mathrm{c}_{p}\left(s_{n}\right)=\mathrm{c}_{p}(p) \Leftrightarrow n\right.$ is even). The increasing strength of $p$ in $\mathrm{P}$ is

$$
\operatorname{Str}_{\text {incr }}(p)= \begin{cases}k_{p} & \text { if } k_{p} \in \omega \text { exists } \\ \omega & \text { otherwise }\end{cases}
$$

Since $\mathrm{P} \in \mathbb{P}_{\text {fin }}$, the latter case can only occur when $p=\perp$. From a gametheoretical viewpoint, if $p \neq \perp$, then $\operatorname{Str}_{\text {incr }}(p)$ corresponds to the length of the strongest $<_{p}$-increasing run that a player can take while playing in $\mathrm{P}$.

In a similar manner, we define the decreasing strength of $p$ in $\mathrm{P}$, denoted by $\operatorname{Str}_{\text {decr }}(p)=k \in \omega$, as the length of the largest strictly $\leq_{p}$-decreasing sequence $\left(s_{n}\right)_{n<k}$ that satisfies $s_{0}=p$ and $\left(c_{p}\left(s_{n}\right)=c_{p}(p) \Leftrightarrow n\right.$ is even). It is well-defined since $\operatorname{Card}(\operatorname{Pred}(p))<\aleph_{0}$ holds for every $p \in P$.

The increasing and decreasing strengths of a node give a good indication of the strength it bears as a position in the game:

Lemma 35. If $\mathrm{P}, \mathrm{Q} \in \mathbb{P}_{\text {fin }}$ and $\tau$ is a winning ultrapositional strategy for II in the game $G_{\mathbb{P}}(\mathrm{P}, \mathrm{Q})$, then for all $p \in P$ :

1. $\operatorname{Str}_{\text {incr }}(p) \leq \operatorname{Str}_{\text {incr }}(\tau(p))$,

2. $\operatorname{Str}_{\text {decr }}(p) \leq \operatorname{Str}_{\text {decr }}(\tau(p))$.

Proof.

1. Towards a contradiction, suppose that $\operatorname{Str}_{\text {incr }}(p)>\operatorname{Str}_{\text {incr }}(\tau(p))$. We proceed by cases.

If $\operatorname{Str}_{\mathbf{i n c r}}(\boldsymbol{p}) \neq \boldsymbol{\omega}$ : assume that $\operatorname{Str}_{\mathrm{incr}}(p)=k$ is witnessed by a sequence $\left(p_{n}\right)_{n<k}$. Since $\tau$ is winning, $\left(\tau\left(p_{n}\right)\right)_{n<k}$ is strictly $\leq_{q}$-increasing and satisfies $\tau\left(p_{0}\right)=\tau(p)$ and $\left(\mathrm{c}_{q}\left(\tau\left(p_{n}\right)\right)=\mathrm{c}_{p}(\tau(p)) \Leftrightarrow n\right.$ is even). Thus $\operatorname{Str}_{\text {incr }}(\tau(p)) \geq k$, a contradiction.

If $\operatorname{Str}_{\text {incr }}(\boldsymbol{p})=\boldsymbol{\omega}$ : for all $k \in \boldsymbol{\omega}$, there exists a strictly $\leq_{p}$-increasing sequence $\left(s_{n}\right)_{n<k}$ that satisfies $s_{0}=p$ and $\left(\mathrm{c}_{p}\left(s_{n}\right)=\mathrm{c}_{p}(p) \Leftrightarrow n\right.$ is even). Since $\tau$ is winning, $\left(\tau\left(p_{n}\right)\right)_{n<k}$ is strictly $\leq_{q}$-increasing and satisfies $\tau\left(p_{0}\right)=\tau(p)$ and $\left(\mathrm{c}_{q}\left(\tau\left(p_{n}\right)\right)=\mathrm{c}_{p}(\tau(p)) \Leftrightarrow n\right.$ is even $)$. Therefore, $\operatorname{Str}_{\text {incr }}(\tau(p))=\omega$, a contradiction.

2. Towards a contradiction, $\operatorname{suppose}$ that $\operatorname{Str}_{\text {decr }}(p)>\operatorname{Str}_{\text {decr }}(\tau(p))$. We also suppose that $\operatorname{Str}_{\text {decr }}(p)=k \in \omega$ is witnessed by a sequence $\left(p_{n}\right)_{n<k}$. Since $\tau$ is winning, $\left(\tau\left(p_{n}\right)\right)_{n<k}$ is strictly $\leq_{q}$-decreasing and satisfies $\tau\left(p_{0}\right)=$

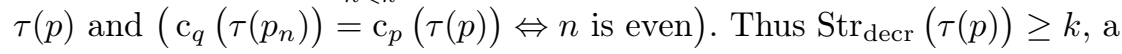
contradiction. 


\section{Ill-foundedness of the Wadge order on the Scott domain}

In this section, we prove that the quasi-order $\leq_{w}$ is already ill-founded inside the class of $\omega$-differences of open sets of the Scott domain.

\section{Theorem 36.}

$$
\left(D_{\omega}\left(\boldsymbol{\Sigma}_{1}^{0}\right)(\mathcal{P} \omega), \leq_{w}\right) \text { is ill-founded }
$$

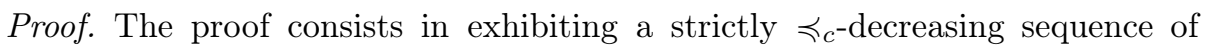
posets $\left(\mathrm{P}_{n}\right)_{n \in \omega^{+}}$in $\mathbb{P}_{\mathrm{emb}}$ and making use of the Lemma 22

First, let us fix $n \in \omega^{+}$. We define $\mathrm{P}_{n}=\left(P_{n}, \leq_{p_{n}}, \mathrm{c}_{p_{n}}\right)$ as the following 2-colored countable poset:

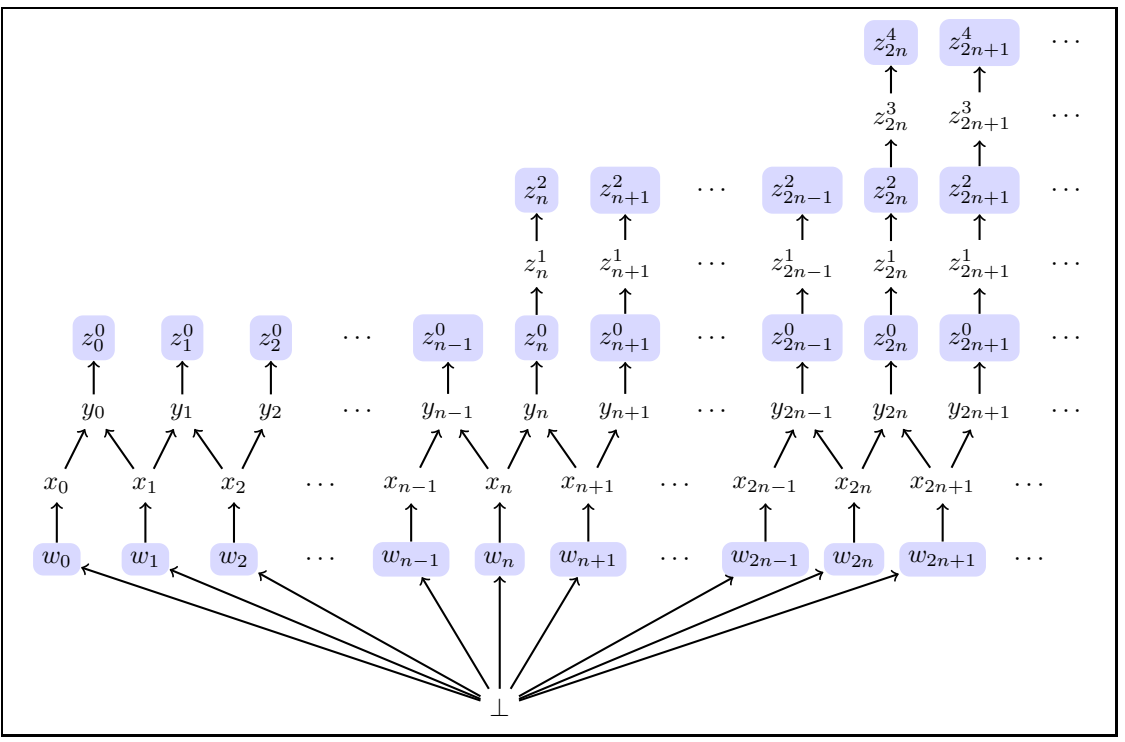

Figure 5: The 2-colored countable poset $\mathrm{P}_{n} \in \mathbb{P}_{\mathrm{emb}}$ for $n \in \omega^{+}$.

Formally, the set of nodes is:

$$
\begin{aligned}
P_{n}= & \{\perp\} \cup\left\{w_{m}, x_{m}, y_{m}\right\}_{m \in \omega} \\
& \cup\left\{z_{m}^{2 k} \mid k \in \omega, n \geq k m\right\} \cup\left\{z_{m}^{2 k+1} \mid k \in \omega, n \geq(k+1) m\right\},
\end{aligned}
$$

the order relation is:

$$
\begin{aligned}
\leq_{p_{n}}= & \left\{\left(\perp, w_{m}\right),\left(w_{m}, x_{m}\right),\left(x_{m}, y_{m}\right),\left(x_{m+1}, y_{m}\right),\left(y_{m}, z_{m}^{0}\right)\right\}_{m \in \omega} \\
& \cup\left\{\left(z_{m}^{k}, z_{m}^{k+1}\right) \mid k \leq\left\lfloor\frac{m}{n}\right\rfloor \cdot 2-1\right\},
\end{aligned}
$$


where $\left\lfloor\frac{m}{n}\right\rfloor$ denotes the integer part of $\frac{m}{n}$, and the 2-coloring is:

$$
\begin{aligned}
\mathrm{c}_{p_{n}}: P_{n} & \rightarrow 2 \\
p & \mapsto 0 \quad \text { if } p \in\left\{\perp, x_{m}, y_{m}\right\}_{m \in \omega} \cup \bigcup_{m \in \omega} z_{m}^{\text {odd }}, \\
p & \mapsto 1 \quad \text { if } p \in\left\{w_{m}\right\}_{m \in \omega} \cup \bigcup_{m \in \omega} z_{m}^{\text {even }},
\end{aligned}
$$

where $z_{m}=\left\{z_{m}^{k} \mid k \leq\left\lfloor\frac{m}{n}\right\rfloor \cdot 2\right\}, z_{m}^{\text {even }}=\left\{z_{m}^{k} \in z_{k} \mid k\right.$ even $\}$, and $z_{m}^{\text {odd }}=\left\{z_{m}^{k} \in\right.$ $z_{k} \mid k$ odd $\}$.

For all $n \in \omega^{+}$, it is easy to check that all the requirements that are needed for $\mathrm{P}_{n}$ to belong to $\mathbb{P}_{\text {fin }}$ are fulfilled. Therefore, by Proposition 33] we have:

$$
\mathcal{A}_{\mathrm{P}_{n}} \in D_{\omega}\left(\boldsymbol{\Sigma}_{1}^{0}\right)(\mathcal{P} \omega) .
$$

For the remainder of the proof, we need some notations. For any $k \in \omega$, we call branch $k$ of $\mathbf{P}_{n}$ the set of nodes $B_{k}=\left\{w_{k}, x_{k}, y_{k}\right\} \cup z_{k}$, and right-shift in $\mathbf{P}_{n}$ any sequence of moves of the form $\left(w_{k}, y_{k}, w_{k+1}\right)$. First, we describe the behavior of an ultrapositional winning strategy facing a right-shift.

Claim 37. Let $n, m \in \omega^{+}$and $\tau$ be an ultrapositional strategy in $G_{\mathbb{P}}\left(\mathrm{P}_{n}, \mathrm{P}_{m}\right)$. If I's moves are a right-shift $\left(w_{k}, y_{k}, w_{k+1}\right)$ and $\tau\left(w_{k}\right) \in B_{l}$ for some $l \in \omega$, then $\tau\left(w_{k+1}\right) \in B_{l^{\prime}}$ for some $l^{\prime} \leq l+1$.

Proof of the claim. We split the proof in two different cases.

If $\boldsymbol{l}=\mathbf{0}$ holds: since $w_{k} \leq_{p_{n}} y_{k}, \mathrm{c}_{p_{n}}\left(y_{k}\right)=0, \tau$ is winning and $\tau\left(w_{k}\right) \in B_{0}$, we get $\tau\left(y_{k}\right) \in\left\{x_{0}, y_{0}\right\}$. Moreover, since $w_{k+1} \leq p_{n} y_{k}, \mathrm{c}_{p_{n}}\left(w_{k+1}\right)=1$ and $\tau$ is winning, we get:

$$
\tau\left(w_{k+1}\right) \in\left\{w_{0}, w_{1}\right\} \subseteq B_{0} \cup B_{1} .
$$

If $\boldsymbol{l} \in \boldsymbol{\omega}^{+}$holds: once again, since $w_{k} \leq_{p_{n}} y_{k}, c_{p_{n}}\left(y_{k}\right)=0, \tau$ is winning and $\tau\left(w_{k}\right) \in B_{l}$, we get $\tau\left(y_{k}\right) \in z_{l-1}^{\text {odd }} \cup z_{l}^{\text {odd }} \cup\left\{x_{l}, y_{l}, y_{l-1}\right\}$. Moreover, since $w_{k+1} \leq_{p_{n}} y_{k}, c_{p_{n}}\left(w_{k+1}\right)=1$ and $\tau$ is winning, we get:

$$
\tau\left(w_{k+1}\right) \in z_{l-1}^{\text {even }} \cup z_{l}^{\text {even }} \cup\left\{w_{l-1}, w_{l}, w_{l+1}\right\} \subseteq \bigcup_{l^{\prime} \leq l+1} B_{l^{\prime}} .
$$

Claim

It remains to show that the sequence $\left(\mathrm{P}_{n}\right)_{n \in \omega_{+}}$is an infinite strictly $\preccurlyeq c^{-}$ decreasing sequence in $\mathbb{P}_{\mathrm{emb}}$.

Claim 38. If $0<n<m<\omega$, then $\mathrm{P}_{m} \preccurlyeq c \mathrm{P}_{n}$.

Proof of the claim. It suffices to observe that $\mathrm{P}_{m}$ is an ideal of $\mathrm{P}_{n}$ and use Proposition 31.

Claim

Claim 39. If $0<n<m<\omega$, then $\mathrm{P}_{n} \aleph_{c} \mathrm{P}_{m}$. 
Proof of the claim. Towards a contradiction, suppose that $\mathrm{P}_{n} \preccurlyeq_{c} \mathrm{P}_{m}$ holds. By Proposition 29, player II has a winning ultrapositional strategy $\tau$ in the game $G_{\mathbb{P}}\left(\mathrm{P}_{n}, \mathrm{P}_{m}\right)$.

The idea of the proof is to construct a particular run of the game that $\tau$ cannot win. By Claim [37, if I plays a sequence of the form $\left(w_{0}, y_{0}, w_{1}, y_{1}, w_{2}, \ldots\right)$ composed with right-shifts, then II's moves are limited. In particular, whenever I shifts from $B_{k}$ to $B_{k+1}$, II can only shift from $B_{l}$ to $B_{l^{\prime}}$ where $l^{\prime} \leq l+1$. Because $n<m$, I can finally reach a node of greater increasing strength than the one reached by II, which leads to a contradiction.

More formally, suppose that I's first move is $w_{0}$ so that $\tau\left(w_{0}\right) \in B_{k_{0}}$ for some $k_{0} \in \omega$, and that I plays a run composed with several right-shifts

$$
\left(w_{0}, y_{0}, w_{1}, y_{1}, w_{2}, \ldots, w_{l}\right) .
$$

By an iteration of Claim 37, we get $\tau\left(w_{l}\right) \in B_{l^{\prime}}$ for some $l^{\prime} \leq k_{0}+l$. Since $n<m$, there exists $n_{0} \in \omega$ such that the following inequalities work:

$$
\operatorname{Str}_{\text {incr }}\left(w_{n m n_{0}}\right)=2 m n_{0}+3>2 n n_{0}+\operatorname{Str}_{\text {incr }}\left(w_{k_{0}}\right) \geq \operatorname{Str}_{\text {incr }}\left(\tau\left(w_{n m n_{0}}\right)\right),
$$

which is a contradiction to Lemma 35 .

Claim

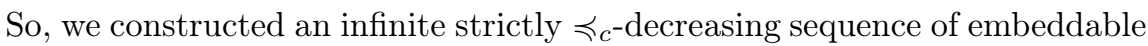
posets, namely

$$
\mathrm{P}_{1} \succ_{c} \mathrm{P}_{2} \succ_{c} \mathrm{P}_{3} \succ_{c} \mathrm{P}_{4} \succ_{c} \ldots
$$

By Lemma 22, we obtain an infinite strictly $\leq_{w}$-decreasing sequence of subsets of $\mathcal{P} \omega$, namely:

$$
\mathcal{A}_{\mathrm{P}_{1}}>{ }_{w} \mathcal{A}_{\mathrm{P}_{2}}>{ }_{w} \mathcal{A}_{\mathrm{P}_{3}}>{ }_{w} \mathcal{A}_{\mathrm{P}_{4}}>{ }_{w} \ldots
$$

which were also proved to be differences of $\omega$ open sets.

\section{Antichains in the Wadge order on the Scott domain}

We prove that infinite $\leq_{w}$-antichains already exist within the class of $\omega$-differences of open subsets of the Scott domain. The proof is nothing but a tailoring of the proof of Theorem 36

\section{Theorem 40.}

$$
\left(D_{\omega}\left(\boldsymbol{\Sigma}_{1}^{0}\right)(\mathcal{P} \omega), \leq_{w}\right) \text { has infinite antichains. }
$$

Proof. We construct an infinite sequence of embeddable posets $\left(\mathrm{Q}_{n}\right)_{n \in \omega_{+}}$that are pairwise $\preccurlyeq c^{- \text {-incomparable. }}$

We fix $n \in \omega^{+}$and define $Q_{n}=\left(Q_{n}, \leq_{q_{n}}, c_{q_{n}}\right)$ as the following 2-colored countable poset: 


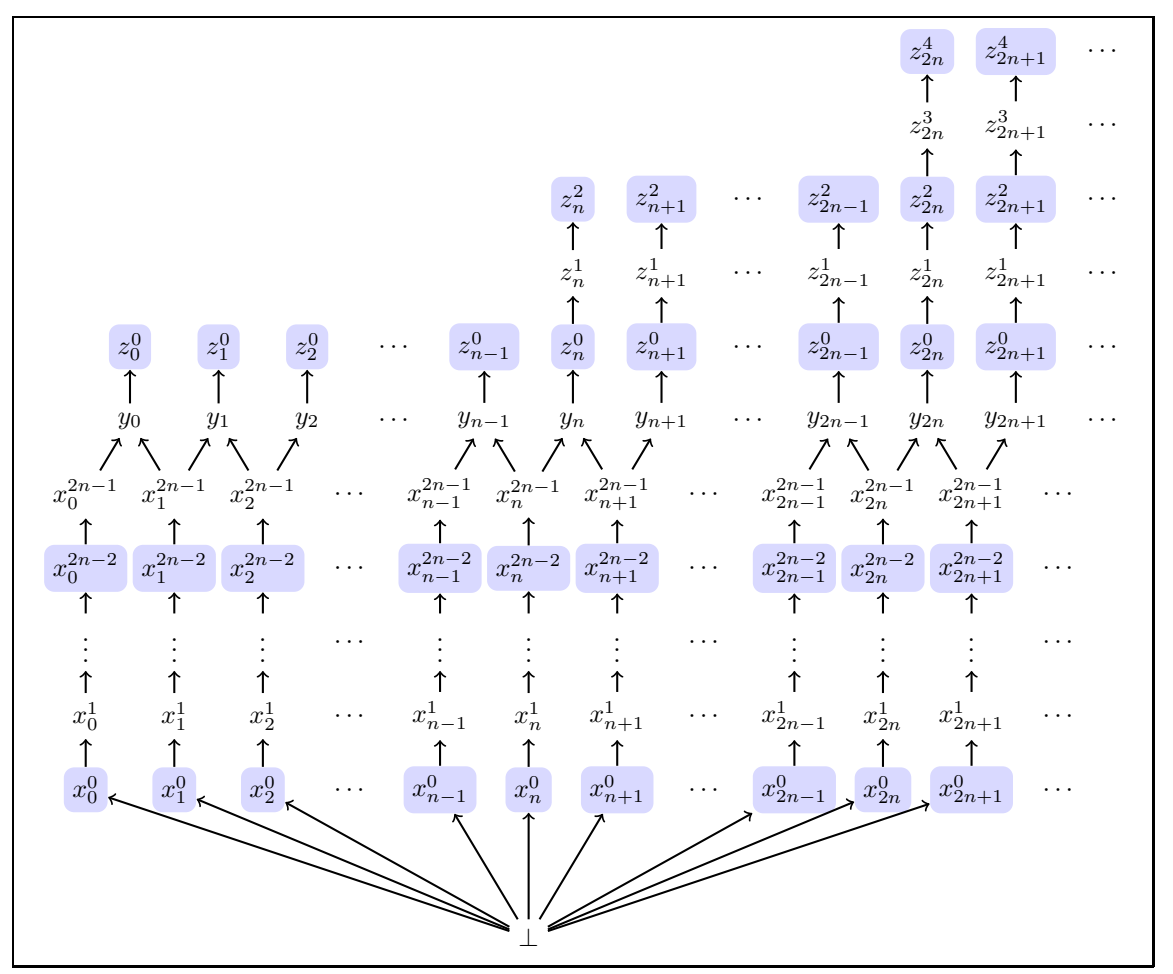

Figure 6: The 2-colored countable poset $\mathrm{Q}_{n} \in \mathbb{P}_{\mathrm{emb}}$ for $n \in \omega^{+}$.

Formally, the set of nodes is:

$$
\begin{aligned}
Q_{n}= & \{\perp\} \cup\left\{x_{m}^{k}, y_{m}\right\}_{m \in \omega, k<2 n} \\
& \cup\left\{z_{m}^{2 k} \mid k \in \omega, n \geq k m\right\} \cup\left\{z_{m}^{2 k+1} \mid k \in \omega, n \geq(k+1) m\right\},
\end{aligned}
$$

the order relation is:

$$
\begin{aligned}
\leq_{q_{n}}= & \left\{\left(\perp, x_{m}^{0}\right),\left(x_{m}^{k}, x_{m}^{k+1}\right),\left(x_{m}^{2 n-1}, y_{m}\right),\left(x_{m+1}^{2 n-1}, y_{m}\right),\left(y_{m}, z_{m}^{0}\right)\right\}_{m \in \omega, k<2 n-1} \\
& \cup\left\{\left(z_{m}^{k}, z_{m}^{k+1}\right) \mid k \leq\left\lfloor\frac{m}{n}\right\rfloor \cdot 2-1\right\},
\end{aligned}
$$

and the coloring is given by the function:

$$
\begin{aligned}
\mathrm{c}_{p_{n}}: P_{n} & \rightarrow 2 \\
p & \mapsto 0 \quad \text { if } p \in\left\{\perp, x_{m}^{2 k+1}, y_{m}\right\}_{m \in \omega, k<n} \cup \bigcup_{m \in \omega} z_{m}^{\text {odd }}, \\
p & \mapsto 1 \quad \text { if } p \in\left\{2_{m}^{2 k}\right\}_{m \in \omega, k<n} \cup \bigcup_{m \in \omega} z_{m}^{\text {even }} .
\end{aligned}
$$

As in the proof of Theorem [36, it is easy to see that $Q_{n} \in \mathbb{P}_{\text {fin }}$, and thus $\mathcal{A}_{\mathrm{Q}_{n}} \in D_{\omega}\left(\boldsymbol{\Sigma}_{1}^{0}\right)(\mathcal{P} \omega)$ holds for every $n \in \omega^{+}$. Now, it remains to show that

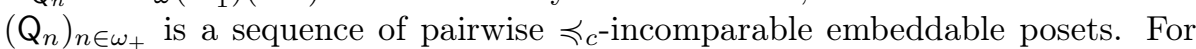
this purpose, we define a right-shift in $\mathrm{Q}_{n}$ as any sequence of moves of the form $\left(x_{k}^{2 n-2}, y_{k}, x_{k+1}^{2 n-2}\right)$ for some $k \in \omega$. 
Claim 41. If $0<n<m<\omega$, then $\mathrm{Q}_{m} \AA_{c} \mathrm{Q}_{n}$.

Proof of the claim. Towards a contradiction, we assume that $\mathrm{Q}_{m} \preccurlyeq_{c} \mathrm{Q}_{n}$ holds. By Proposition 29, II has an ultrapositional winning strategy $\tau$ in the game $G_{\mathbb{P}}\left(\mathrm{Q}_{m}, \mathrm{Q}_{n}\right)$.

The idea of the proof is to exhibit some specific run for I in this game that $\tau$ cannot beat. For this purpose, player I will use the fact that $n<m$ and several right-shifts to reach an element $q \in \mathrm{Q}_{n}$ which has a larger increasing strength than $\tau(q)$.

We consider $x_{0}^{2 m-2}$ as I's first move. If II's first move is $x_{i}^{2 j}$ for some $i \in \omega$ and $j<n$, then $\operatorname{Str}_{\text {decr }}\left(x_{0}^{2 m-2}\right)=2 m>2 n \geq \operatorname{Str}_{\text {decr }}\left(x_{i}^{2 j}\right)$, which contradicts Lemma [35. Since $c_{q_{m}}\left(x_{0}^{2 m-2}\right)=1$, we can assume that $\tau\left(x_{0}^{2 m-2}\right)=z_{l_{0}}^{2 k}$ for some $k, l_{0} \in \omega$.

If I's second move is $y_{0}$, then II's second move has color 0 . Hence, II's second move is of the form $z_{l_{0}}^{2 k^{\prime}+1}$ for some $k^{\prime} \in \omega$.

Since $\operatorname{Str}_{\text {decr }}\left(x_{1}^{2 m-2}\right)=2 m>2 n \geq \operatorname{Str}_{\text {decr }}\left(x_{i}^{2 j}\right)$ for all $j<n$, if I's third move is $x_{1}^{2 m-2}$, then Lemma 35 implies that II's third move cannot be of the form $x_{i}^{2 j}$ for some $i, j \in \omega$. So, II's third move is of the form $z_{l_{0}}^{2 k^{\prime \prime}}$ for some $k^{\prime \prime} \in \omega$.

Now, consider the run where I plays right-shifts:

$$
\left(x_{0}^{2 m-2}, y_{0}, x_{1}^{2 m-2}, y_{1}, x_{2}^{2 m-2}, y_{2}, \ldots\right) .
$$

By the previous observations, II will only play in $z_{l_{0}}$. But there exists $i_{0} \in \omega$ such that

$$
\operatorname{Str}_{\text {incr }}\left(y_{i_{0}}\right)>\max \left\{\operatorname{Strincr}_{\text {inc }}(q) \mid q \in z_{l_{0}}\right\},
$$

which contradicts Lemma 35

Claim

For the last two claims, we need to introduce the notion of branches in $\mathrm{Q}_{n}$. For any $k \in \omega$, we call branch $k$ of $\mathbf{Q}_{n}$ the set of nodes $B_{k}=\left\{x_{k}^{l}, y_{k}\right\}_{l<2 n} \cup z_{k}$. The next claim, which concerns the 2-colored countable posets of the form $Q_{n}$ for some $n \in \omega^{+}$, is a tailoring of Claim 37 .

Claim 42. Let $n, m \in \omega^{+}$and $\tau$ be an ultrapositional strategy in $G_{\mathbb{P}}\left(\mathrm{Q}_{n}, \mathrm{Q}_{m}\right)$. If I's moves are a right-shift $\left(x_{k}^{2 n-2}, y_{k}, x_{k+1}^{2 n-2}\right)$ and $\tau\left(x_{k}^{2 n-2}\right) \in B_{l}$ holds for some $l \in \omega$, then $\tau\left(x_{k+1}^{2 n-2}\right) \in B_{l^{\prime}}$ holds for some $l^{\prime} \leq l+1$.

Proof of the claim. We proceed as in the proof of Claim 37 except that the right-shift $\left(w_{k}, y_{k}, w_{k+1}\right)$ in $\mathbf{P}_{n}$ is replaced by the right-shift $\left(x_{k}^{2 n-2}, y_{k}, x_{k+1}^{2 n-2}\right)$ in $Q_{n}$.

$\square$ Claim

With the help of the previous claim, we finally obtain:

Claim 43. If $0<n<m<\omega$, then $\mathrm{Q}_{n} \aleph_{c} \mathrm{Q}_{m}$.

Proof of the claim. We proceed as in the proof of Claim 39. Towards a contradiction, suppose that $\mathrm{Q}_{n} \preccurlyeq_{c} \mathrm{Q}_{m}$ holds. By Proposition 29] player II has a winning ultrapositional strategy $\tau$ in the game $G_{\mathbb{P}}\left(\mathrm{Q}_{n}, \mathrm{Q}_{m}\right)$. 
Suppose that I's first move is $x_{0}^{2 n-2}$ so that $\tau\left(x_{0}^{2 n-2}\right) \in B_{k_{0}}$ for some $k_{0} \in \omega$, and that I plays a run composed with several right-shifts

$$
\left(x_{0}^{2 n-2}, y_{0}, x_{1}^{2 n-2}, y_{1}, x_{2}^{2 n-2} \ldots, x_{l}^{2 n-2}\right) .
$$

By an iteration of Claim 42, we get $\tau\left(x_{l}^{2 n-2}\right) \in B_{l^{\prime}}$ for some $l^{\prime} \leq k_{0}+l$. Since $n<m$, there exists $n_{0} \in \omega$ such that the following inequalities work:

$$
\operatorname{Str}_{\text {incr }}\left(x_{n m n_{0}}^{2 n-2}\right)=2 m n_{0}+3>2 n n_{0}+\operatorname{Str}_{\text {incr }}\left(x_{k_{0}}^{0}\right) \geq \operatorname{Str}_{\text {incr }}\left(\tau\left(x_{n m n_{0}}^{2 n-2}\right)\right),
$$

which contradicts Lemma 35

$\square$ Claim

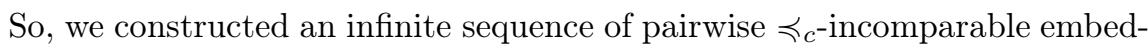
dable posets, namely $\left(Q_{n}\right)_{n \in \omega^{+}}$. By Lemma 22, we obtain an infinite sequence of pairwise $\leq{ }_{w}$-incomparable subsets of $\mathcal{P} \omega$, namely $\left(\mathcal{A}_{\mathrm{Q}_{n}}\right)_{n \in \omega^{+}}$. We also proved that all these sets are $\omega$-differences of open sets.

\section{Open questions}

We conclude with some related open questions that may serve as guidelines for future work.

In Theorem 26, we exhibited a partial order on a class of 2-colored countable posets which embeds in the Wadge order on the $\boldsymbol{\Delta}_{2}^{0}$-degrees of $\mathcal{P} \omega$. It would be desirable to find a better description of this partial order, as it was recently done in KM17 for the Baire space $\omega^{\omega}$ - the space of infinite sequence of integers endowed with the product of the discrete topology. More precisely, they showed that the Wadge order on the Borel subsets of $\omega^{\omega}$ can be represented by countable joins of countable transfinite nests of well-founded trees labeled by 2. Although such a description seems to be out of reach for the whole Borel subsets, a reasonable question would be:

Question 1. Is there any standard order-theoretic structure which is isomorphic to $\left(\boldsymbol{\Delta}_{2}^{0}(\mathcal{P} \omega), \leq_{w}\right) / \equiv_{w}$ ?

We showed that some unwanted properties already occur at a very low topological complexity level in the Wadge order of $\mathcal{P} \omega$. By looking at some reductions that are more general than the continuous ones, these bad behaviors may disappear. For example, Motto Ros, Schlicht and Selivanov consider the class of $\boldsymbol{\Sigma}_{\omega}^{0}$-functions $\mathcal{F}_{0}=\left\{f: \mathcal{P} \omega \rightarrow \mathcal{P} \omega: f^{-1}(\mathcal{A}) \in \boldsymbol{\Sigma}_{\omega}^{0}(\mathcal{P} \omega)\right.$ for any $\left.\mathcal{A} \in \boldsymbol{\Sigma}_{\omega}^{0}(\mathcal{P} \omega)\right\}$ MRSS15. They show that $\leq_{\mathcal{F}_{0}} 11$ induces a well-quasi-order on the Borel subsets of $\mathcal{P} \omega$. Thus, the following question seems of interest:

Question 2. For which classes of functions $\mathcal{F} \subseteq \mathcal{F}_{0}$ containing the continuous ones is the induced order $\leq_{\mathcal{F}}$ on the Borel subsets of $\mathcal{P} \omega$ a well-quasi-order?

Another relevant question concerns the possibility of extending our results to some other quasi-Polish spaces. We essentially focused on $\mathcal{P} \omega$ because it is universal among them. Since we showed that $\mathcal{P} \omega$ is not well-behaved with respect to the Wadge order, one may ask where the well-behaved quasi-Polish spaces may be found.

\footnotetext{
${ }^{11}$ We write $\mathcal{A} \leq \mathcal{F}_{0} \mathcal{B}$ if there exists $f \in \mathcal{F}_{0}$ such that $f^{-1}[\mathcal{B}]=\mathcal{A}$.
} 
Question 3. Is there a natural characterization of the quasi-Polish spaces whose Wadge order on the Borel subsets is a well-quasi-order?

In the metrizable setting, Schlicht proved that the Polish spaces for which $\leq_{w}$ is a well-quasi-order on the Borel subsets are exactly the zero-dimensional ones [Sch18]. It would be interesting to know whether this property somehow extends to the quasi-Polish spaces.

\section{References}

[AL12] Alessandro Andretta and Alain Louveau, Wadge degrees and pointclasses: Introduction to Part III, In [KLS12], 2012, pp. 3-23.

[And07] Alessandro Andretta, The SLO principle and the Wadge hierarchy, Foundations of the formal sciences V, Stud. Log. (Lond.), vol. 11, Coll. Publ., London, 2007, pp. 1-38.

[BG15] Verónica Becher and Serge Grigorieff, Wadge hardness in Scott spaces and its effectivization, Math. Structures Comput. Sci. 25 (2015), no. 7, 1520-1545.

[dB13] Matthew de Brecht, Quasi-Polish spaces, Ann. Pure Appl. Logic 164 (2013), no. 3, 356-381.

[Dup01] Jacques Duparc, Wadge hierarchy and Veblen hierarchy. I. Borel sets of finite rank, J. Symbolic Logic 66 (2001), no. 1, 56-86.

$\left[\mathrm{GHK}^{+} 03\right]$ G. Gierz, K. H. Hofmann, K. Keimel, J. D. Lawson, M. Mislove, and D. S. Scott, Continuous Lattices and Domains, Encyclopedia of Mathematics and its Applications, vol. 93, Cambridge University Press, Cambridge, 2003.

[GL13] Jean Goubault-Larrecq, Non-Hausdorff topology and domain theory, New Mathematical Monographs, vol. 22, Cambridge University Press, Cambridge, 2013.

[Ike10] Daisuke Ikegami, Games in Set Theory and Logic, 2010, Ph.D. Thesis, Universiteit van Amsterdam, Amsterdam.

[Kec95] Alexander S. Kechris, Classical descriptive set theory, Graduate Texts in Mathematics, vol. 156, Springer-Verlag, New York, 1995.

[KLS12] Alexander S. Kechris, Benedikt Löwe, and John R. Steel (eds.), Wadge degrees and projective ordinals. The Cabal Seminar. Volume II, Lecture Notes in Logic, vol. 37, Association for Symbolic Logic, La Jolla, CA; Cambridge University Press, Cambridge, 2012.

[KM17] Takayuki Kihara and Antonio Montalbán, On the structure of the Wadge degrees of BQO-valued Borel functions, arXiv preprint arXiv:1705.07802 (2017).

[Lou12] Alain Louveau, Some results in the Wadge hierarchy of Borel sets, In [KLS12, 2012, pp. 47-73. 
[LSR12] Alain Louveau and Jean Saint-Raymond, The strength of Borel Wadge determinacy, In [KLS12, 2012, pp. 74-101.

[MRSS15] Luca Motto Ros, Philipp Schlicht, and Victor Selivanov, Wadgelike reducibilities on arbitrary quasi-Polish spaces, Math. Structures Comput. Sci. 25 (2015), no. 8, 1705-1754.

[Peq15] Yann Pequignot, A Wadge hierarchy for second countable spaces, Arch. Math. Logic 54 (2015), no. 5-6, 659-683.

[Sch18] Philipp Schlicht, Continuous reducibility and dimension of metric spaces, Arch. Math. Logic 57 (2018), no. 3-4, 329-359.

[Sco76] Dana Scott, Data types as lattices, SIAM J. Comput. 5 (1976), no. 3, $522-587$.

[Sel05] Victor L. Selivanov, Hierarchies in $\phi$-spaces and applications, MLQ Math. Log. Q. 51 (2005), no. 1, 45-61.

[Sel06] - Towards a descriptive set theory for domain-like structures, Theoret. Comput. Sci. 365 (2006), no. 3, 258-282.

[VW12] Robert Van Wesep, Wadge degrees and descriptive set theory, In KLS12, 2012, pp. 24-42.

[Wad83] William W. Wadge, Reducibility and determinateness on the Baire space, 1983, Ph.D. Thesis, University of California, Berkeley.

[Wad12] - Early investigations of the degrees of Borel sets, In [KLS12], 2012, pp. 166-195.

[Wei00] Klaus Weihrauch, Computable analysis: An introduction, Texts in Theoretical Computer Science. An EATCS Series, Springer-Verlag, Berlin, 2000. 